\title{
Deep analysis of wild Vitis flower transcriptome reveals unexplored genome regions associated with sex specification
}

\author{
Miguel Jesus Nunes Ramos $^{1} \cdot$ João Lucas Coito ${ }^{1}$ Joana Fino ${ }^{2} \cdot$ Jorge Cunha $^{3,4}$. \\ Helena Silva ${ }^{5}$ - Patrícia Gomes de Almeida ${ }^{6}$ - Maria Manuela Ribeiro Costa ${ }^{5}$. \\ Sara Amâncio ${ }^{1} \cdot$ Octávio S. Paulo $^{2} \cdot$ Margarida Rocheta $^{1} \mathbb{D}$
}

Received: 29 March 2016 / Accepted: 15 October 2016 / Published online: 24 October 2016

(C) Springer Science+Business Media Dordrecht 2016

\begin{abstract}
Key message RNA-seq of Vitis during early stages of bud development, in male, female and hermaphrodite flowers, identified new loci outside of annotated gene models, suggesting their involvement in sex establishment.

Abstract The molecular mechanisms responsible for flower sex specification remain unclear for most plant species. In the case of $V$. vinifera ssp. vinifera, it is not fully understood what
\end{abstract}

Miguel Jesus Nunes Ramos and João Lucas Coito have contributed equally to this work.

Electronic supplementary material The online version of this article (doi:10.1007/s11103-016-0553-9) contains supplementary material, which is available to authorized users.

Margarida Rocheta

rocheta@isa.ulisboa.pt

1 Universidade de Lisboa, Instituto Superior de Agronomia, LEAF, Linking Landscape, Environment, Agriculture and Food, Tapada da Ajuda, 1359-017 Lisboa, Portugal

2 Computational Biology and Population Genomics Group, Centre for Ecology, Evolution and Environmental Changes, Faculdade de Ciências, Universidade de Lisboa, 1749-016 Lisboa, Portugal

3 Instituto Nacional de Investigação Agrária e Veterinária, Quinta d'Almoinha, Dois Portos, Portugal

4 Instituto de Tecnologia Química e Biológica, Universidade Nova de Lisboa, Oeiras, Portugal

5 BioSystems \& Integrative Sciences Institute (BioISI), Plant Functional Biology Centre, University of Minho, Campus de Gualtar, 4710-057 Braga, Portugal

6 Development and Evolutionary Morphogenesis, Centre for Ecology, Evolution and Environmental Change, Faculdade de Ciências, Universidade de Lisboa, 1749-016 Lisboa, Portugal determines hermaphroditism in the domesticated subspecies and male or female flowers in wild dioecious relatives (Vitis vinifera ssp. sylvestris). Here, we describe a de novo assembly of the transcriptome of three flower developmental stages from the three Vitis vinifera flower types. The validation of de novo assembly showed a correlation of 0.825 . The main goals of this work were the identification of $V . v$. sylvestris exclusive transcripts and the characterization of differential gene expression during flower development. RNA from several flower developmental stages was used previously to generate Illumina sequence reads. Through a sequential de novo assembly strategy one comprehensive transcriptome comprising 95,516 non-redundant transcripts was assembled. From this dataset 81,064 transcripts were annotated to $V$. $v$. vinifera reference transcriptome and 11,084 were annotated against $V$. v. vinifera reference genome. Moreover, we found 3368 transcripts that could not be mapped to Vitis reference genome. From all the non-redundant transcripts that were assembled, bioinformatics analysis identified 133 specific of $V$. v. sylvestris and 516 transcripts differentially expressed among the three flower types. The detection of transcription from areas of the genome not currently annotated suggests active transcription of previously unannotated genomic loci during early stages of bud development.

Keywords Vitis vinifera $\mathrm{ssp}$. sylvestris · RNA-seq · De novo assembly - Differential gene expression · Sex loci

\section{Introduction}

Transcriptome analysis is an important tool for the characterization and understanding of the molecular basis of phenotypic diversity between individuals. Thus, among the 
complex genetic mechanisms controlling plasticity, sex differentiation can be interpreted from the dynamics of transcriptome. High throughput RNA-sequencing analysis of highly complex transcriptomes, can now be applied to any tissue despite the developmental stage (Wang et al. 2009).

Vitis vinifera $\mathrm{ssp}$. vinifera is the domesticated grapevine, a hermaphrodite plant and one of the most valuable cultivated fruit crop in the world, with 67 tons of grapes produced in 2012 (http://faostat.fao.org/). On the other hand, Vitis wild relative, Vitis vinifera ssp. sylvestris, has separate sexes, and is a repository of genetic diversity, essential for the maintenance of genetic variability and to limit genetic erosion of the domesticated variety (García and Revilla 2013). As distinctive feature male flowers present a reduced pistil without style or stigma and female flowers have reflexed stamens with infertile pollen. The development of a flower requires the synchronization of gene activities, environmental conditions and hormones (de Montaigu et al. 2010; Huijser and Schmid 2011). The decision to make a flower encloses sex-specific signals that activate or repress maleor female-specific pathways leading to the differentiation of reproductive organs. Among these signals, plant hormones play an important role. They are involved in various aspects of reproductive organ development in hermaphrodite plants but also in plants with unisexual flowering, such as monoecious and dioecious plants (Lebel-Hardenack and Grant 1997).

Several studies have shown the effects of hormones on sexual specification (Golenberg and West 2013; Manzano et al. 2014; Sekhar and Sawhney 1991). Interestingly, hormone effects are plant species dependent; there are no merely masculinizing or feminizing hormones. For instance, gibberellic acid has a masculinizing effect in cucumber and Asparagus officinalis (Fuchs et al. 1977; Lazarte and Garrison 1980) whereas a feminizing effect is observed in maize (Dellaporta and Calderon-Urrea 1994). Also, auxin has a feminizing effect in Opuntia stenopetala (Orozco-Arroyo et al. 2012) but a masculinizing effect in Mercurialis annиa (Durand and Durand 1991), while ethylene has a feminizing role in cucurbit flower development (Boualem et al. 2008) but a masculinizing effect in watermelon (Manzano et al. 2014). Other plant hormones like abscisic acid that have a feminizing effect in Solanum carolinense and jasmonate with a masculinizing action in maize also contribute to flower sexual differentiation (Browse 2009; Hartwig et al. 2011). In addition, cytokinins have been related to feminized the phenotype in Asparagus officinalis (Lazarte and Garrison 1980) and Vitis vinifera (Negi and Olmo 1966).

Additionally, previous studies identify a putative locus for flower sex in $V . v$. vinifera in chromosome 2 (chr2) (Dalbó et al. 2000; Lowe and Walker 2006; Marguerit et al. 2009), located in a region that comprise a few genes that may play a role in hormone metabolism (Battilana et al.
2013). Other authors using BAC clones established a physical map of $143 \mathrm{~kb}$ (Fechter et al. 2012). Sequence and annotation of this region identified 11 genes possibly responsible for flower sex determination. The most promising of these genes, an ADENINE PHOSPHORIBOSYL TRANSFERASE $(A P T)$, became a marker able to distinguish the female allele from the male/hermaphrodite alleles (Fechter et al. 2012). More recently a new study gave a more detailed insight regarding a possible sex locus in chr2 of $V$. v. vinifera suggested a region of $152 \mathrm{~kb}$ that comprising the previously referred $143 \mathrm{~kb}$ region and included several genes with XY type polymorphism (Picq et al. 2014). All these studies reinforce the existence of a locus in chr2 responsible for sex determination in Vitis v. vinifera. However, in the most recent Vitis annotation (12X_v2.1) (http://genomes. cribi.unipd.it/grape/), some genes in the $152 \mathrm{~kb}$ region are absent from the annotation or are located in the chromosome Unknown.

Our aim is to map and annotate unexplored transcripts and to get a list of genes with differential expression all along early flower development of both Vitis vinifera subspecies through a new assembly of Vitis transcriptome. We reason that some of the undiscovered transcripts may play important roles in Vitis sex differentiation. To achieve our goal we de novo assembled the Illumina RNA-seq raw data of flower Vitis transcriptome previous obtained (Ramos et al. 2014). The assembly of the transcriptome data of three different stages of flower development from each of the three Vitis flower types (hermaphrodite, male and female) produced a non-negligible fraction of unmapped contigs, whose sequences are generally neglected in favor of the mapped contigs despite potentially containing useful information. This analysis of transcriptome dynamics takes advantage of the of RNA-seq approach to discover transcribed loci outside of annotated gene models, as well as genes differentially expressed during early Vitis flower development.

\section{Materials and methods}

\section{Raw data source and strategy of sample analysis}

Previous transcriptome sequencing of flower buds in early in early developmental stages was made through RNA-seq of flower buds from Vitis vinifera sylvestris female and male plants and from the hermaphrodite Vitis vinifera vinifera $\mathrm{cv}$. Touriga Nacional [TN] as previously described (Ramos et al. 2014). Floral buds were collected during the month of April (2012) from a single plant of each flower type and classified according to its developmental stage, B, D, G and $\mathrm{H}$ (Baggiolini 1952), and treated separately, adding to a total of twelve samples (Ramos et al. 2014). In order to 
evaluate data consistency, two Illumina independent runs were carried out on each sample. The raw data generated in the previous work was used to perform a new assembly. However, in this new assembly, we decided to deep analyze three stages B, D and $\mathrm{H}$. The exclusion of stage $\mathrm{G}$ from the analysis was due an unusual lower number of reads in the male flower, comparatively to the other stages and flower types, which could mask the results of differential expression gene analysis, a constrain not present in the previous analysis (Ramos et al. 2014).

\section{Transcriptome de novo assembly and annotation}

cDNA libraries construction and transcriptome sequencing were performed by BaseClear, B.V, Netherlands (Ramos et al. 2014) and quality assessment was performed using SeqtrimNEXT (Falgueras et al. 2010). The input configuration was set to remove indeterminations, poly-A-tails, Illumina adapters, contaminant sequences, vector residues, low quality zones and repetitive/complex regions (Online Resource 1).

The nine datasets were assembled in a unique transcriptome in order to obtain a more robust result than with individual assemblies using the multiple k-mer method. Assemblies with k-mers of 21, 25, 29 and 31 were performed with Velvet package (Zerbino 2010): reads prepared with velveth, with options -fastq and -short were assembled with velvetg with option -read trkg. Corrections were applied with Oases (Schulz et al. 2012) using the ideal cut-off (0.2) suggested by Velveth Optimizer (Victorian Bioinformatics Consortium). The final merge was performed with the same programs with a k-mer of 31, as suggested by Velvet Optimizer as the ideal k-mer. To reduce redundancy and group transcripts, our library was submitted to CD-HIT-EST algorithm (Li and Godzik 2006). CD-HIT-EST works by clustering sequences according to their similarity and outputs the larger sequence of each contig, although some redundancy persisted after CD-HIT-EST. A pipeline based on BLAST was then used to deal with this issue. A blast of our transcriptomic library against itself was conducted with blastn version 2.2.28 (Altschul et al. 1997) with an e-value limit of 1e-5. An in-house python script was used to remove redundant and small contigs, considering $90 \%$ of identity which is available upon request. Finally, contigs were matched against the NCBI databases to exclude potential contaminations (e.g. microorganisms).

In order to evaluate the assembly, in addition to N50 calculation, we ran RSEM-EVAL from DETONATE (Li et al. 2014) with the same raw data files and with the non-redundant fasta sequences previously assembled, considering an average of $100 \mathrm{bp}$ per read.

Genome, transcriptome and annotation versions $12 \mathrm{X}$ v2.1 of Vitis vinifera vinifera cv. PN40024 were downloaded from Grape Genome Database on CRIBI (http://genomes. cribi.unipd.it/grape/). Non-redundant libraries were blasted on Vitis $v$. vinifera reference transcriptome (Altschul et al. 1997), with an e-value limit of 1e-5. A second in-house python script to map and rename each aligned contig was developed. Reads were classified as exonic if they mapped to an annotated exon; intronic, if they mapped within the $5^{\prime}$ and 3' boundaries of a given transcript, but outside annotated exons; or intergenic, if they aligned outside known boundaries of annotated genes. In order to get accurate data only contigs with length equal or higher to $150 \mathrm{bp}$ (Celaj et al. 2014) were kept. The remaining sequences were discarded, as they reduce our ability to identify meaningful confident sequence matches. For each sample its expression

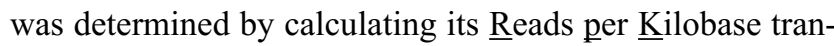
script per Million reads (RPKM) for each library described below.

The non-aligned contigs were blasted to Vitis $v$. vinifera reference genome with the same parameters described above. To map contigs to the reference genome, three different approaches were applied. On the first approach only the contigs that mapped to a gene were considered; on the second approach, contigs mapping within $1000 \mathrm{bp}$ upstream or downstream of a gene were considered as being part of that gene; finally, on the third approach, the flanking gene regions were increased to $4000 \mathrm{bp}$ upstream or downstream of the annotated gene (Zenoni et al. 2010). Sequences and its flanking regions were manually inspected and the second approach was considered the best. Reads that mapped inside this region were assigned as unannotated exons or untranslated regions (UTRs), while reads that mapped outside these regions were denominated as putatively novel transcripts. Additionally, only contigs with RPKM equal or higher than one were kept.

Finally, a third python script was used to map and rename sequences that aligned to the genome. All in-house programs are available upon request.

Moreover, cis association on adjacent genes was also studied on all sequences that mapped successfully to the reference transcriptome and genome. Sequences associated to unknown or X-random chromosomes were excluded, since it was not possible to correctly infer the real localization of the genes in the chromosome. Moreover, in order to reduce false positive associations, only transcripts with expression correlations higher than 0.8 were considered.

In addition to Vitis reference genome, the unmapped sequences were blasted against seven databases present in Ensemble (Arabidopsis thaliana, Medicago truncatula, Oryza sativa, Populus trichocarpa, Solanum lycopersicum, Solanum tuberosum and Zea mays - http://plants.ensembl. org) considering an e-value limit of 1e-5. The sequences that did not map with these databases were considered putative contaminations or novel genes. A general blast (e-value 
limit of 1e-5) against all NCBI database was performed and sequences from bacteria, fungi and similar organisms were discarded as contaminants.

Contigs that did not map in databases were split into sequences of $100 \mathrm{bp}$ and each sequence treated separately. These fragmented sequences were blasted against the NCBI nt database. This strategy allowed the identification of chimeric sequences that could not be mapped previously. Additionally, a self-blast was applied to these $100 \mathrm{bp}$ sequences in order to confirm the similarity to other unmapped sequences. For these two blasts an e-value cutoff of 1e-5 was considered. The remaining sequences were considered as "completely unknown" and were submitted to Repbase (http://www.girinst.org/), with default parameters, in order to predict if these sequences belong to mobile elements.

\section{Transcriptome de novo assembly validation and gene expression reproducibility through RT-qPCR}

To determine the accuracy of the de novo assembly, a correlation was established between the de novo assembly and the previous one (Ramos et al. 2014) (Online Resource 2). For gene validation through RT-qPCR, four genes with annotation ABNORMAL FLORAL ORGANS (VIT_202s0154g00070), FLAVIN-CONTAININGMONOOXYGENASE-LIKE(VIT_20 2s0154g00170), FLAVIN-CONTAINING MONOOXYGENASE LIKE-3 (VIT_202s0154g00180) and AGAMOUSLIKE 19 (AGL 19; VIT_202s0025g04650) related to flower development present in chromosome 2 were used (Online Resources 3, 4). Additionally, some of the completely unknown transcripts (G 30546; G 80794; G 89407) were validated in order to determine if their expression is maintained in new biological samples collected in 2015 (Online Resources 3, 4).

The cDNA concentration in RT-qPCR was achieved through a serial of decimal dilutions from 2.1 to $0.021 \mu \mathrm{g}$ of cDNA. Amplification reactions were performed in triplicates of $20 \mu \mathrm{L}$ containing $5 \mu \mathrm{L}$ of master mix (SsoFast EvaGreen Supermix, Bio-Rad, Hercules, CA), $0.4 \mu \mathrm{M}$ of specific primers, $0.21 \mu \mathrm{g}$ of cDNA and autoclaved water. The following program was applied: initial polymerase activation, $95^{\circ} \mathrm{C}, 2 \mathrm{~min}$; then 40 cycles at $95^{\circ} \mathrm{C}, 15 \mathrm{~s}$ (denaturation); $57^{\circ} \mathrm{C}, 30 \mathrm{~s}$ (annealing); $76^{\circ} \mathrm{C}, 30 \mathrm{~s}$ (extension) with a single fluorescence reading taken at the end of each cycle. Each run was completed with a melting curve analysis to confirm the specificity of amplification and the lack of primer dimmers. To confirm amplicon size, products were run on $1.7 \%(\mathrm{w} / \mathrm{v})$ agarose gels.

RT-qPCR runs were perform, for each gene, with a series of decimal dilutions of a precisely calculated number of plasmid copies (3000-3 pg) to create a calibration ruler. Cqs (threshold cycles) obtained from RNA samples was matched against the calibration ruler to estimate the copy number of each transcript on samples. The absolute copy number was calculated using the following formula: Copy number $=\mathrm{C} \times \mathrm{NA} / \mathrm{M}$; where Copy number, number of molecules $/ \mu \mathrm{L}$ contained in the purified cDNA; $\mathrm{C}$, concentration of the purified cDNA $(\mathrm{g} / \mu \mathrm{L}) ; \mathrm{M}$, the molecular weight of the cDNA gene fragment; NA, Avogadro's number $=6.023 \times 1023$ molecules $/$ mole. The coefficient of correlation ( $\mathrm{r}$ ) between de novo assembly samples and RTqPCR were also calculated.

\section{Differentially expressed genes (DEGs)}

The number of RPKM was quantified and assumed as an expression value. RPKMs were calculated individually for each sample, matching the cleaned reads library from each sample to the total assembly contigs. This assessment was achieved using RSEM package (Kelley et al. 2012). Contigs file was formatted using rsem-prepare-reference algorithm and quantification was performed with rsem-calculate-expression. A mismatch of three nucleotides was permitted (optionbowtie-n 3). The analysis of differential gene expression was also performed with RSEM package (Li and Dewey 2011). The data matrix was exported from the rsem-calculate-expression output, using rsem-generate-data-matrix algorithm. The analysis itself was performed by rsem-run-ebseq algorithm.

The transcripts falsely detected as differentially expressed were corrected using rsem-control-fdr algorithm with a false discovery rate (FDR) of 0.05 . Fold change was manually filtered according its $\log _{2}$, in Excel (Microsoft). Transcripts with $\log _{2}$ between -1 and 1 were considered false positives and discarded. Additionally, in the absence of a control sample that could be used as a reference for all samples, DEGs in female and male flowers were determined using the hermaphrodite as a reference, while the male transcriptome was used as a reference when female and male were compared. We opted for the use of "up-expressed" and "downexpressed" for differential gene expression when comparing two flower transcriptomes. In addition to the previous filters applied, for the expression analysis we only considered contigs with RPKM equal or higher than 1 to be expressed.

\section{Results and discussion}

\section{Transcriptome de novo assembly and validation}

One of the objectives of this work was to identify exclusive and unidentified transcripts sequences with a putative role in sex specification in Vitis vinifera sylvestris by comparison with Vitis vinifera vinifera (Touriga Nacional) and the reference genome (Vitis vinifera vinifera $\mathrm{cv}$. PN40024). To achieve this purpose, a de novo assembly of Vitis vinifera transcripts was performed (described in "Materials and methods" section). 
From our previous study (Ramos et al. 2014), we used $368,898,213$ sequence reads of 45-50 bp covering $15-17 \mathrm{~Gb}$ of sequence data. Each flower developmental stage was represented by an average of 30 million reads (Online Resource 1). The trimming/filtering with SeqtrimNEXT removed $6 \%$ of the initial reads, and the remaining 345,641,042 reads from the nine samples were pooled and assembled, resulting in 231,342 contigs (Table 1 ). After removing redundancy, only $41 \%(95,516)$ of the contigs remained, with an average size of $390 \mathrm{bp}$. The N50 statistic of 887 bp was used in further analysis (Table 1). N50 is a quality measure value, which describes the point of hall mass of our data, meaning that $50 \%$ of the sequenced bases were assembled into contigs with this length or higher (Zerbino and Birney 2008). Also, the program RSEM-EVAL from DETONATE (Li et al. 2014) was used to evaluate the reliability of the assembly. The assembly was scored as - 754,768,755, which is not a comparable value to other studies since RSEM-EVAL score is dependent of the number of reads found on a RNA-seq library (Li et al. 2014). In order to get a clear support to this assembly, the "contig impact score" was evaluated to each contig. This parameter is an indication that the contig is supported by the original reads. On average, the contigs produced on this assembly were scored as 1487, which, by being a positive value, provides confidence on this assembly ( $\mathrm{Li}$ et al. 2014).

To determine the accuracy of the de novo assembly, a correlation with the previous RNA-seq was established

Table 1 New assembly parameters and raw results of nine transcriptomes, developmental stages $\mathrm{B}, \mathrm{D}$ and $\mathrm{H}$, from $V$. v. sylvestris flowers (male and female) and $V$. v. vinifera (hermaphrodite)

\begin{tabular}{ll}
\hline Assembly parameters & \\
Optimal k-mer & 31 \\
Optimal cut-off & 0.2 \\
Obtained contigs & \\
Obtained & 231,342 \\
After CD-HITS-EST & 177,105 \\
Non redundant & 95,516 \\
Blast to $V$. v. vinifera transcriptome (cDNA) & \\
Mapped & \\
Total & 81,064 \\
One hit & 28,466 \\
More hits & 52,598 \\
Unmapped & 14,452 \\
Blast to $V . v$ vinifera genome (DNA) & \\
Mapped & \\
Total & 11,084 \\
One hit & 8161 \\
More hits & 2923 \\
Unmapped & 3368 \\
\hline
\end{tabular}

${ }^{\mathrm{a}}$ Contigs that have no match with the $V$. v. vinifera transcriptome have been mapped against $V$. v. vinifera genome version $12 \mathrm{X} \_\mathrm{v} 2.1$
(Ramos et al. 2014). Data showed a correlation of $r=0.825$, which guaranteed the quality of data comparison (Online Resource 2). However, the expression of some transcripts is relatively different in the comparison of assemblies. This situation results from improvements done to the reference transcriptome. Initially, when all reads were mapped against the reference genome, there was only one isoform described per gene (version 12X_v1) (Ramos et al. 2014). As a consequence each gene was evaluated only by one RPKM value. The most recent transcriptome annotation (12X_v2.1) has multiple isoforms assigned to each gene and allows assigning a RPKM value to each isoform. The match between both assemblies translates differences in the expression of different isoforms of the same gene. Despite these specific situations, the correlation between both assemblies is high and positive, proving that the de novo assembly is consistent with the previous one (Ramos et al. 2014).

Additionally, gene expression validation was performed through RT-qPCR with seven transcripts and the correlation between RT-qPCR and a de novo assembly shows and $r$ between 0.989 and 0.831 which confirm data precision (Online Resource 4).

\section{Mapping and annotation to Vitis reference transcriptome and genome}

The mapping of non-redundant contigs against the Vitis vinifera vinifera reference transcriptome allowed us to predict the role of $85 \%$ of the contigs that matched with Vitis reference transcriptome $(81,064)$ (Table 1$)$. The contigs that did not map to the reference transcriptome were then blasted to the reference genome and $12 \%$ of the total was successfully mapped $(11,084)$ (Table 1$)$ in intronic or intergenic regions. The remaining 3368 contigs did not map to the Vitis available genome (Table 1). When redundancy was removed former numbers decreased but the highest proportion of contigs (72\%) map to previously annotated exons of the Vitis transcriptome with $7 \%$ of the reads aligning to intronic regions or UTRs, $18 \%$ aligning outside of the boundaries of annotated genes, i.e. intergenic areas and $3 \%$ are considered as unmapped sequences (Fig. 1). This could be due to the active transcription of not yet annotated genes or, alternatively, to the genomic differences (such as polymorphisms) between the samples and the reference genome or even to the potential erroneous base calling by the sequencing technology (Oshlack et al. 2010). Additionally, such sequences might also represent non-coding RNAs, repetitive sequences such as mobile elements or miRNAs precursor (pre-miRNA) playing a role in Vitis flower development.

In order to predict possible loci regulated by cis associations, the expression of sequences that unambiguously match to the Vitis reference genome and transcriptome was 


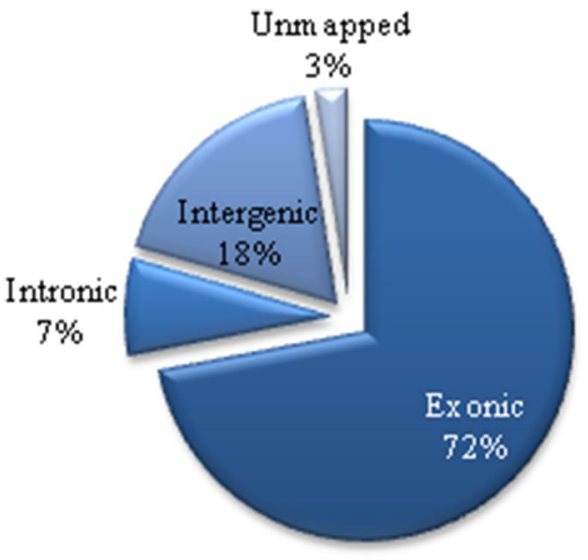

Fig. 1 Overview of sequences transcribed in the genomes in three developmental $V$. vinifera flower stages. The Vitis data used to perform annotation was 12X_v2.1. Redundancy has been removed and only contigs with sequence length $\geq 150 \mathrm{bp}$ were considered

analyzed. To perform this analysis some sequences were discarded: those that did not map to the reference genome, since it was not possible to predict which transcripts were adjacent; and sequences that mapped to the unknown or random chromosomes, as those are virtual chromosomes where the position of transcripts was arbitrarily assigned. Overall, from the 21,745 considered transcripts, there are 688 loci where two or more adjacent transcripts show strong positive $(>0.8)$ co-expression, comprehending a total of 1479 transcripts (Table 2). Only one locus was composed by six adjacent transcripts putatively related, located on the chromosome 12 (chr12:1,628,012-1,630,325). These transcripts are short (157-253 bps) and expressed only in hermaphrodite flowers at developmental stages $\mathrm{D}$ and $\mathrm{H}$. The first transcript found in this cluster (chr12:1,628,012-1,628,238) was successfully blasted to a non-coding RNA, predicted by automated computational analysis, but with no additional information regarding its function (NCBI accession,

Table 2 Summary of putative cis-associated transcripts. Cluster size, represents the number of transcripts that form the cluster; loci count, indicates the number of loci found with strong positive $(>0.8)$ gene expression association and the total number of transcripts represent the total number of transcripts associated

\begin{tabular}{rlr}
$\begin{array}{l}\text { Loci } \\
\text { count }\end{array}$ & $\begin{array}{l}\text { Cluster size (no } \\
\text { of associated } \\
\text { transcripts) }\end{array}$ & $\begin{array}{l}\text { Total number of } \\
\text { transcripts (loci } \\
\text { count } \times \text { cluster } \\
\text { size) }\end{array}$ \\
\hline 607 & 2 & 1214 \\
64 & 3 & 192 \\
13 & 4 & 52 \\
3 & 5 & 15 \\
1 & 6 & 6 \\
688 & & 1479
\end{tabular}

XR 786969.1). For the remaining five transcripts on this cluster, this is the first time that they are reported as being actively transcribed, still with unknown function.

\section{Expression analysis in exonic, intronic and intergenic regions}

The number of transcripts that map to the exonic regions of Vitis annotated gene (Vitis reference transcriptome) increases during flower development in the three flower types (Fig. 2a). The female, male and hermaphrodite flower have an increase of expressed genes of 2, 1.9 and 3.8\%, respectively, from early stage $B$ to late stage $\mathrm{H}$ (Fig. 2a).

The number of the transcripts that align to intronic regions does not follow the same pattern as the exonic transcripts (Fig. 2a). The transcriptome of the female, male and hermaphrodite flower increase $19.4,1.7$ and $11.9 \%$ respectively, from early stage B to late stage H (Fig. 2a). However, the female flower shows a slight decrease in stage D, while at the same stage the male one presents an increase of expressed genes. As a whole, this dynamic highlight the requirement of the hermaphrodite flower type to has more active genes for sex specification in later flower developmental stages. Interestingly, there is increased gene activity in intronic regions. In the male flower, the levels of transcription in intronic regions do not always mirror the pattern of exonic regions across flower developmental stages (Fig. 2a). Our data point that changes in intronic expression directly measure changes in transcriptional activity, a fact reported in other works (Gaidatzis et al. 2015; Sultan et al. 2008). Additionally, it has been pointed that intronic expression are a stand-in for nascent transcription (Ameur et al. 2011; Hendriks et al. 2014). We do not rule out the hypothesis that this systematic study in early flower development mimics what has been reported for mammals development (Hogenesch and Ueda 2011; Mohawk et al. 2012) and in Vitis flower development there is a rhythmic balance between exonic and intronic gene expression, however, still unknown in its function.

The Venn diagram allows to compare the number of exclusive and shared genes between the three flower types, in each flower developmental stage (Fig. 2b). It is possible to observe that the number of expressed common genes to the three flower types increases during flower development. The number of exclusive transcripts in the female drops at stage $\mathrm{D}$ increasing in the stage $\mathrm{H}$; the male shows a progressive decrease in exclusive transcripts as flower development proceeds; the number of genes exclusively expressed in hermaphrodites does not change significantly throughout flower development (Fig. 2b). At later stages (H) female and hermaphrodite flowers share the highest number of transcripts, which could be an indication that similar biological pathways are taking place. Notice that a lower 


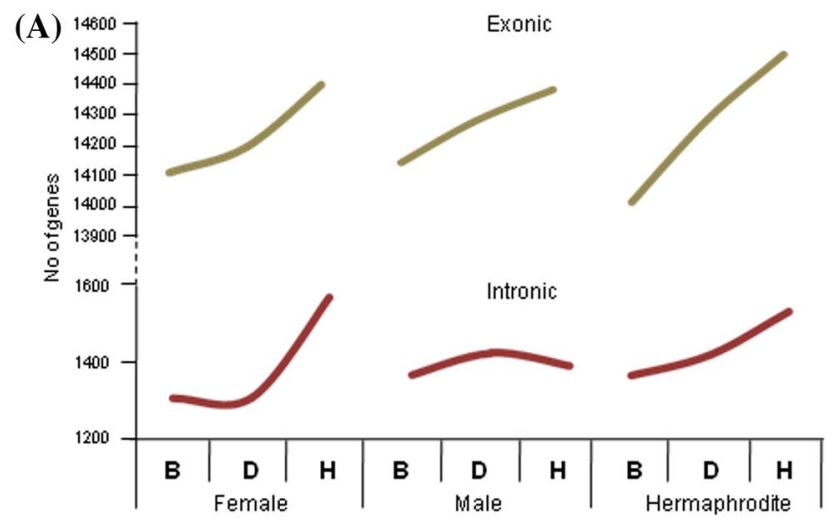

(B)

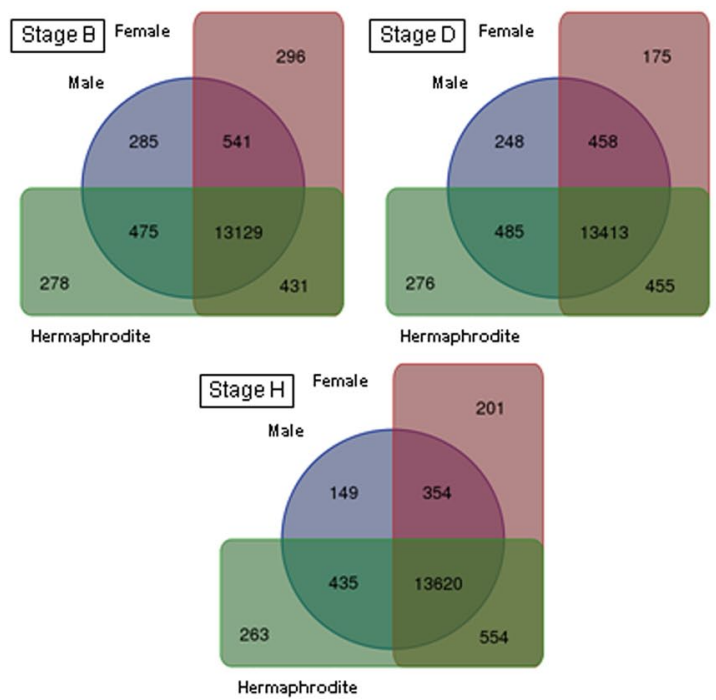

Fig. 2 Analysis of genes transcribed in the genomes that match with $V$. $v$ vinifera reference transcriptome and genome. a Graphical tendency of the number of sequences transcribed in exonic and intronic regions between grapevine flower developmental stages and flower types. b Venn diagrams with the number of genes expressed in each flower developmental stage (B, D and $\mathrm{H})$. Redundancy has been removed and only contigs with RPKM $\geq 1$ and sequence length $\geq 150$ bp were considered

number of expressed genes were obtained when compared with our previous work (Ramos et al. 2014), mainly due to the straight filters applied to get an accurate analysis. Additionally, the new assembly allowed the identification of a significant number of contigs that map to intergenic regions (Fig. 3a). In general, an increase in the number of these unidentified expressed genes is observed throughout flower development for all flower types. However, the stage $\mathrm{H}$ is the one that presents more expressed genes in female and hermaphrodite transcriptome. The number of transcripts in male flower samples increases along development, particularly in the early stages of flowering; however, fewer transcripts from intergenic regions are present in the stage $\mathrm{H}$ when compared with the two other flower types (Fig. 3a). This result highlights the presence of polyadenylated transcripts from presumed intergenic regions with a different dynamic in male flower compared with the female and hermaphrodite. The occurrence of intergenic transcription point out for the possibility that there are more functional genes yet to be discovered. But we cannot exclude the possibility that these regions could drive expression in genes (Bondino and Valle 2009; Moghe et al. 2013) involved in flower development. However, it is not yet clear whether these transcripts represent novel non-coding or protein-coding genes, or the importance of their role during Vitis flower development.

The analysis of transcripts mapped in the intergenic regions that are shared or gender exclusive shows fluctuations between the developmental stages (Fig. 3b). Notice that the number of genes shared between female ( $V . v . s y l$ vestris) and the hermaphrodite $(V . v$ vinifera) it is higher when compared with other flower types and increase across development which suggest a higher proximity between the development of these two types of flowers then between female and male (V.v. sylvestris).

One advantage of RNA-seq is the possibility to detect the expression of non-annotated genomic loci, allowing the detection of novel transcripts. Such areas, initially dubbed as transcribed "dark matter" (Ponting and Belgard 2010), have been the subject of increasing attention. Our results contribute to the increased recognition of the phenomenon of non-exonic, "dark matter" transcription (Kapranov and St Laurent 2012). The non-exonic transcription could be an effect of alternative splicing that lead to the inclusion of new exons not yet annotated.

The high proportion of non-exonic reads may also be explained by uncharacterized gene expression specific to Vitis flower development. Taken together, our data suggest that the development of a Vitis flower is associated with an increase in expression of intronic and intergenic regions. Such regions may represent novel exons, or genes whose inclusion into mature transcripts is induced during flower development. These genes would thus be previously undescribed in Vitis tissues, and are therefore not included in the Vitis transcriptome annotation.

\section{Expression analysis in unexplored Vitis regions}

The remaining contigs that did not map to $V$. $v$. vinifera reference transcriptome nor to the reference genome named "unmapped" were blasted against NCBI databases that include other works on $V$. v. vinifera besides the reference (http://genomes.cribi.unipd.it/grape/). Depending on the blast results, those contigs were divided in three classes: (I) mapped to other Vitis species, (II) homologous to other plant species genes or (III) "completely unknown" if they did not have homology with any sequence present in databases.

Moreover, considering that the existence of completely unknown sequences can result from chimeras, an artifact 


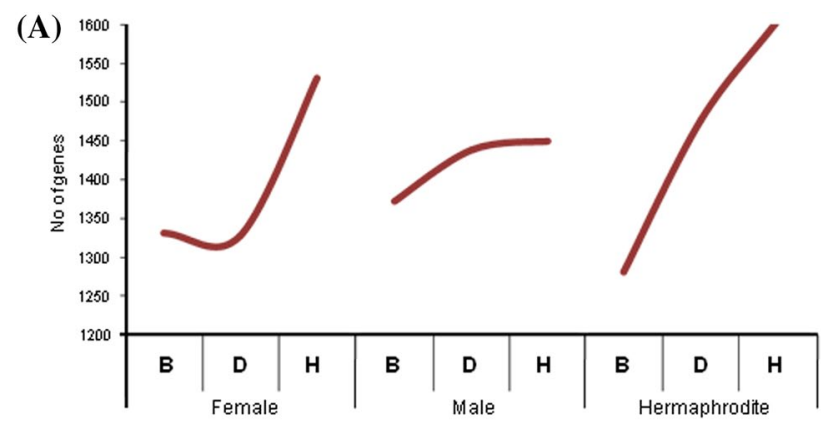

(B)

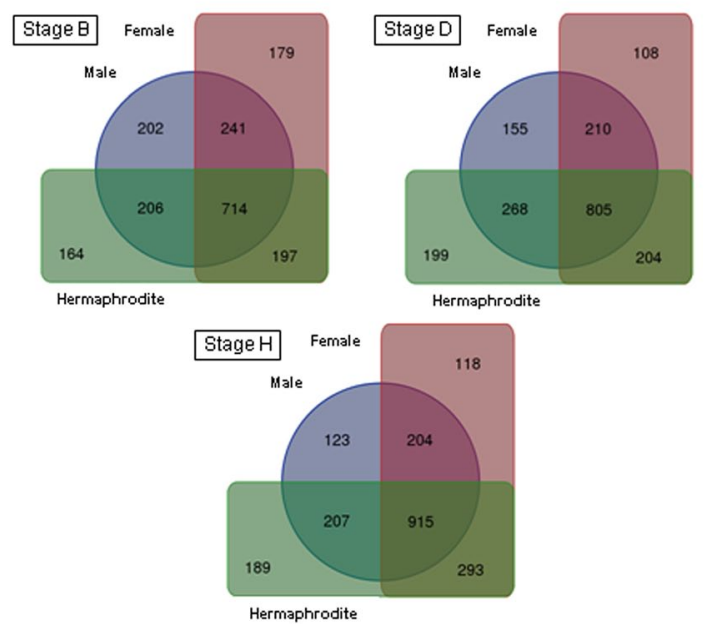

Fig. 3 Analysis of intergenic sequences transcribed in the genomes that match with $V$. v. vinifera reference genome. a Graphical tendency of the number of sequences transcribed between grapevine flower developmental stages and flower types. b Venn diagrams with the number of exclusive intergenic sequences, as well as, sequences shared by flower types, in each flower developmental stage (B, D and H). Only contigs with RPKM $\geq 1$ and sequence length $\geq 150$ bp were considered

that could lead to false sequences, the 1286 completely unknown transcripts were split into $100 \mathrm{bp}$, originating 3081 sequences that were treated individually and subjected to two different blastn approaches. A blastn was performed against all NCBI nt database, to predict sequences that had not map previously. On a second approach, the split sequences were blasted against themselves, in order to identify possible self and multi-gene chimeras. Using the first approach, 67 out of 1286 contigs had one or more sequences with positive results against NCBI nt database. This outcome suggests that some of the original completely unknown sequences had not been properly blasted, perhaps because they were too large. A blast of each short sequence against all the 3081 transcripts reveals situations where contigs were formed by the successive repetition of the same reads. This approach revealed eight putative chimeras (four self and other four multi-gene), but only three were found using both strategies simultaneously. A total of 72 identified chimeras were removed from further analysis, while 1214 completely unknown transcripts remained and were considered. Additionally, to reinforce that those "completely unknown" sequences are Vitis transcripts and not contaminants or artifacts created on the assembly process, three of them (G_30546; G_80794; G_89407) were amplified in cDNA samples from the three flower types collected in different years, using the same phenological developmental stage B (Online Resource 5). The amplification of each one of these transcripts resulted in a clear fragment, with the expected size in different flower types and sampling years. Overall, these results confirm that the completely unknown transcripts are not a result of chimeras.

The number of transcripts that mapped to $V$. $v$. vinifera and those that map against other plant species have a slight variation among flower types and flower developmental stages (Fig. 4a). Conversely, the number of contigs with no homology to any database drops during flower development in each gender (Fig. 4a).

Following the dynamics of these "completely unknown" transcripts shared between flower types during flower development shows a male flower with a lower percentage of exclusive transcripts as the development proceeds, while female and hermaphrodite flowers have an opposite behavior (Fig. 4b). In addition, the higher percentage of expressed genes shared between female and male flowers in stage B is a good indication that similar processes are taking place at this stage in both flower types. On the other hand, female and hermaphrodite flowers shared the highest percentage of expressed genes in the later flower developmental stage (stage H). A profile of these "completely unknown" transcripts (Da Silva et al. 2013) shows some clusters of promising transcripts that could have a role in sex specification (Fig. 5). Novel gene information from Vitis flower transcriptomes can be used for further gene expression studies, to elucidate and understanding molecular processes related with flower development and to gain a deeper functional annotation of Vitis genome and transcriptome.

\section{DEGs analysis}

Gene expression of specific genes may affect flower sex specification, not only due to tissue specificity but also to differential transcription levels. Therefore, we analyzed the differential gene expression between flower types along flower developmental stages. It is clear that the majority of genes with differential expression are found in the earlier stage (B) and in the later one (H) (Fig. 6). When male and female samples are compared with the hermaphrodite, the majority of genes differentially expressed at stage B are found in the male sample. At the same stage, the comparison between male and female transcriptomes shows female with less up-expressed 
Fig. 4 Analysis of transcribed sequences that do not match with Vitis reference genome (12X_v2.1). a Number of transcripts that mapped with $V$. $v$. vinifera outside the reference genome annotation (orange); genes that mapped with other plant species (blue) and genes that not mapped against any known database (brown). b Venn diagrams with the percentage and number of "completely unknown" transcripts shared by flower types, in each flower developmental stage (B, D and $\mathrm{H})$. Filters applied were: $\mathrm{RPKM} \geq 1$ and sequence length $\geq 150 \mathrm{bp}$

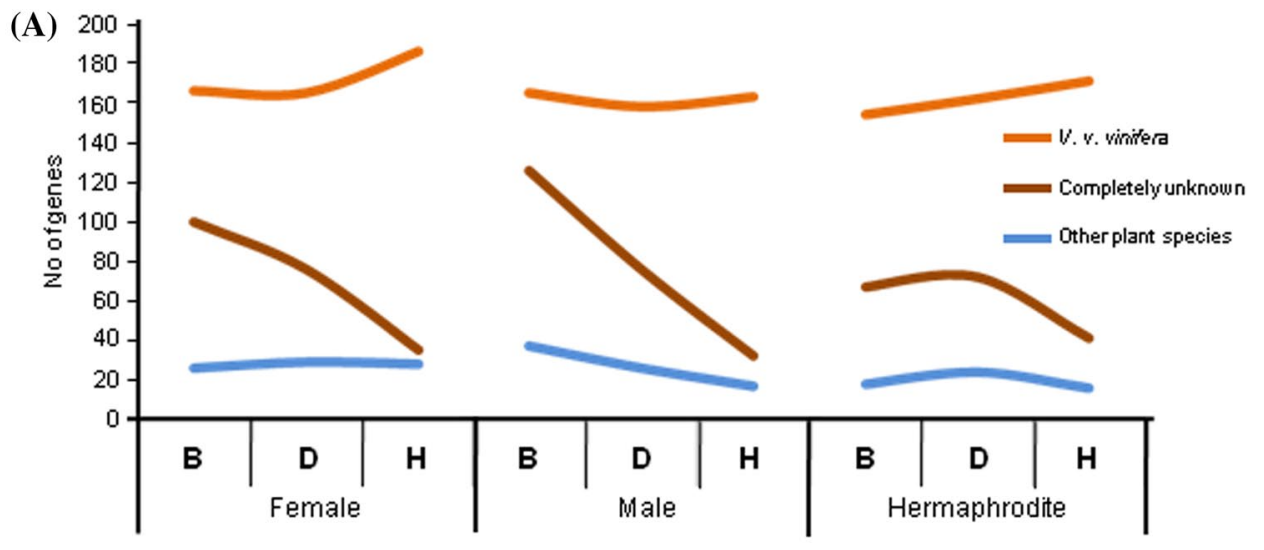

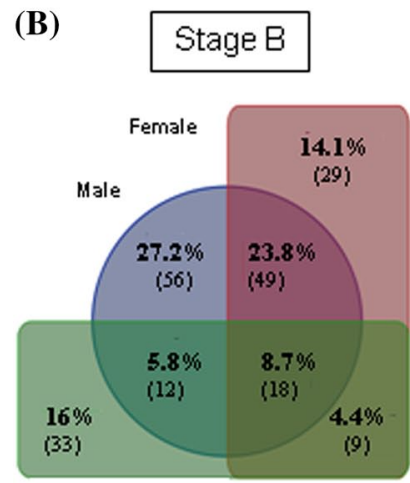

Hermaphrodite

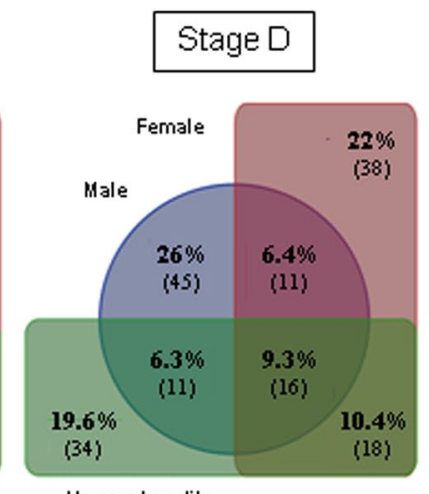

Hermaphrodite

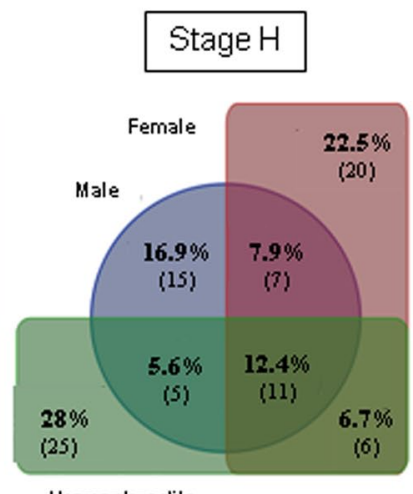

Hermaphrodite genes (Fig. 6). At stage D, the number of differentially expressed genes decreases in all samples while at developmental stage $\mathrm{H}$, the highest number of DEGs was found in female when compared to the hermaphrodite (Fig. 6). At the same stage the number of up- and downexpressed genes is similar in male and hermaphrodite flower (Fig. 6). Taking the results as a whole an opposite dynamics between male and female flowers development is evident across flower development. Male flowers in the initial phase of flower development need more DEGs, while the female flower requires more DEGs in the last flower stage analyzed (stage $\mathrm{H}$ ). This behavior translates differences between sexes and may include unknown mechanisms suppressing carpel development (early) in male flowers, or preventing the correct formation of male organs in female plants (late). The D stage where rudimentary leaves start to appear still collected in a rosette (Baggiolini 1952), transcription slowed in all three types of flowers for some reason not yet known. Our results are in agreement with a previous study applied to Arabidopsis thaliana (Ryan et al. 2015), where temporal gene expression was analyzed during different flowering phases. This work reinforces an extensive view of gene expression activities between flower types during developmental stages.

\section{Differentially expressed genes assigned to gene functional categories}

The data reveal that most DEGs belong to the Primary Metabolism category, followed by the Transport Overview category with second highest number of DEGs detected (Fig. 7; Online Resource 6). Nevertheless, when analyzing the percentage of number of genes relative to the total number of annotated genes in each category, the highest percentage of DEGs is found in the Cellular Metabolism, Secondary Metabolism and Response to Stimulus categories (Fig. 7). Notice that in the Development functional category, when comparing male and hermaphrodite flowers, male has the highest percentage of up-expressed genes in stage $\mathrm{B}$, but then shows more down-expressed genes in the later developmental stages. In the female flower, the number of upexpressed genes is almost constant until stage $\mathrm{H}$, where it shows the highest percentage. In this category no DEGs were observed between female and male transcriptome in early stage $\mathrm{B}$ while in development stage $\mathrm{H}$ the female flower has no down-expressed genes when compared with the male or hermaphrodite transcriptome (Fig. 7). At individual level we analyzed several genes, present in the Development functional category, differentially expressed between flower types. These genes are reported to be important and relevant 


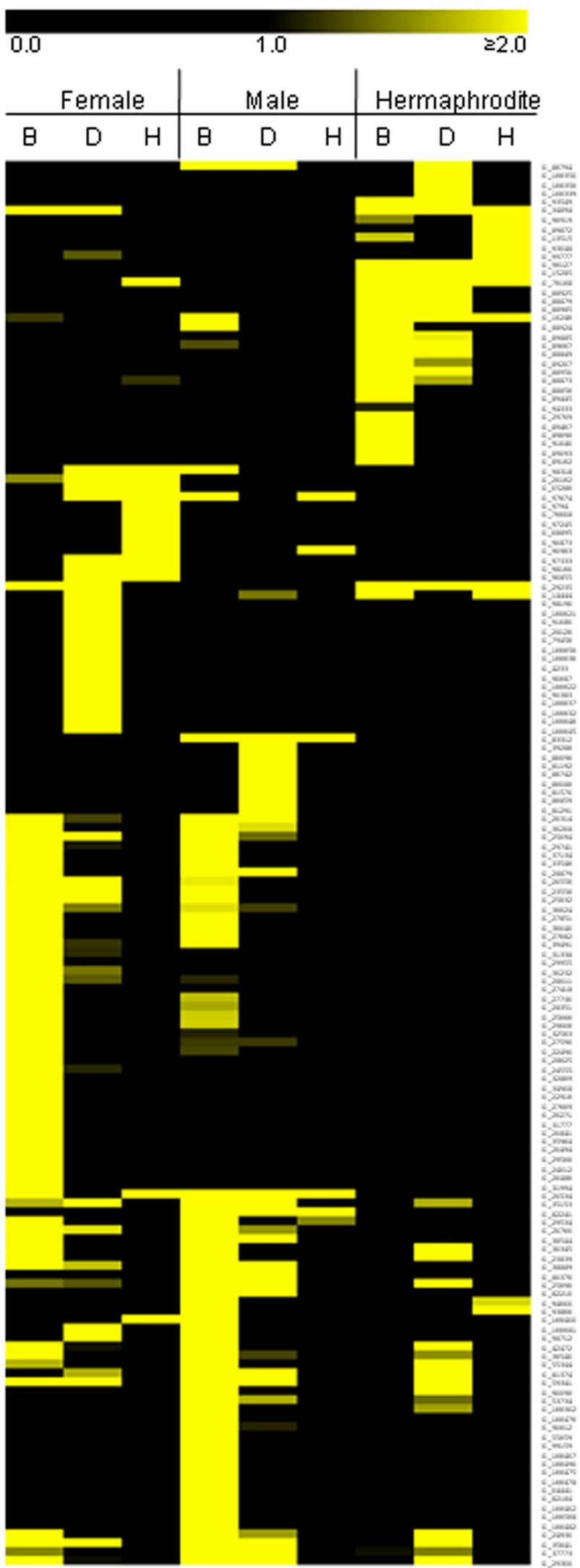

4 Fig. 5 Expression profile of Unknown transcripts. Expression profile of completely unknown transcripts present in the three Vitis flower types in development stages B, D and H. The color profile translates the expression value measured in RPKM

in flower sex development. Among these genes, VviSPLAYED (VviSYD - VIT_205s0020g02000) is required for flower development and patterning (Wagner and Meyerowitz 2002). Its interaction with $L E A F Y$ and SEPALLATA3 regulate the activation of $A P 3$ and $A G$ during flower development (Wagner and Meyerowitz 2002). Additionally, $S Y D$ is also an activator of $W U S$ and required for the maintenance of shoot apical meristem (Kwon et al. 2005; Wu et al. 2012). In our work VviSPLAYED is down-regulated in development stage B in male plants when compared with hermaphrodite plants in the same development stage. However, there are no obvious differences in gene expression in subsequent stages and phenotypically male plants bloom early than female or hermaphrodite plants. This might indicate a slight delay in male flower development at initial stages. Other gene is Flowering Time Control gene (VviFCA VIT_205s0077g00710). FCA gene is a RNA-binding protein that promotes microRNA172 gene activity which in turn represses $A P 2$ from the forth whorl during flower development (Aukerman and Sakai 2003; Jung et al. 2012). Our analysis show that male plants in development stage $\mathrm{H}$ display a down-expression of $V v i F C A$ when compared with female and hermaphrodite plants. This down-regulation of $V v i F C A$ might be relevant taking into consideration the abortion of carpel structures in late development stages in male plants. However, no differences were detected between the three flower types in the expression of the gene (VIT 206s0061g00290) that codes for microRNA172 which suggest another $V v i F C A$ regulation in Vitis. A relevant set of genes with differential expression is NODULIN MtN3, from the Lipoxygenase family that plays an important role in exine pattern determination in microspores (Guan et al. 2008). This gene was covered in our previous work (Ramos et al. 2014) and in this new assembly VviNODULIN MtN3 is, again, highly up-expressed in development stage $\mathrm{H}$ of female plants when compared with both male and hermaphrodite plants in the same development stage. Female plants display infertile pollen with and oval shape and without pores (Gallardo et al. 2009). This up-regulation of VviNODULIN MtN3 might play a role in excessive deposition of exine in female pollen making it infertile and thus contributing to the feminizing phenotype in Vitis vinifera sylvestris female plants. It is not to exclude that key player(s) for sex specification in Vitis might be located in the Unknown functional category, in the current Vitis annotation. The differential expression analysis of this functional category shows 
Fig. 6 Differentially expressed genes between flower types in each developmental stage. DEGs in female and male were calculated by using the hermaphrodite transcriptome as reference. DEGs between female and male, the male transcriptome was the reference. Filters used: $\mathrm{FDR}=0.05$ and $-1<\log 2$ (fold change) $>1, F$ female, $M$ male, Her hermaphrodite. $(*)$ Sample used as reference

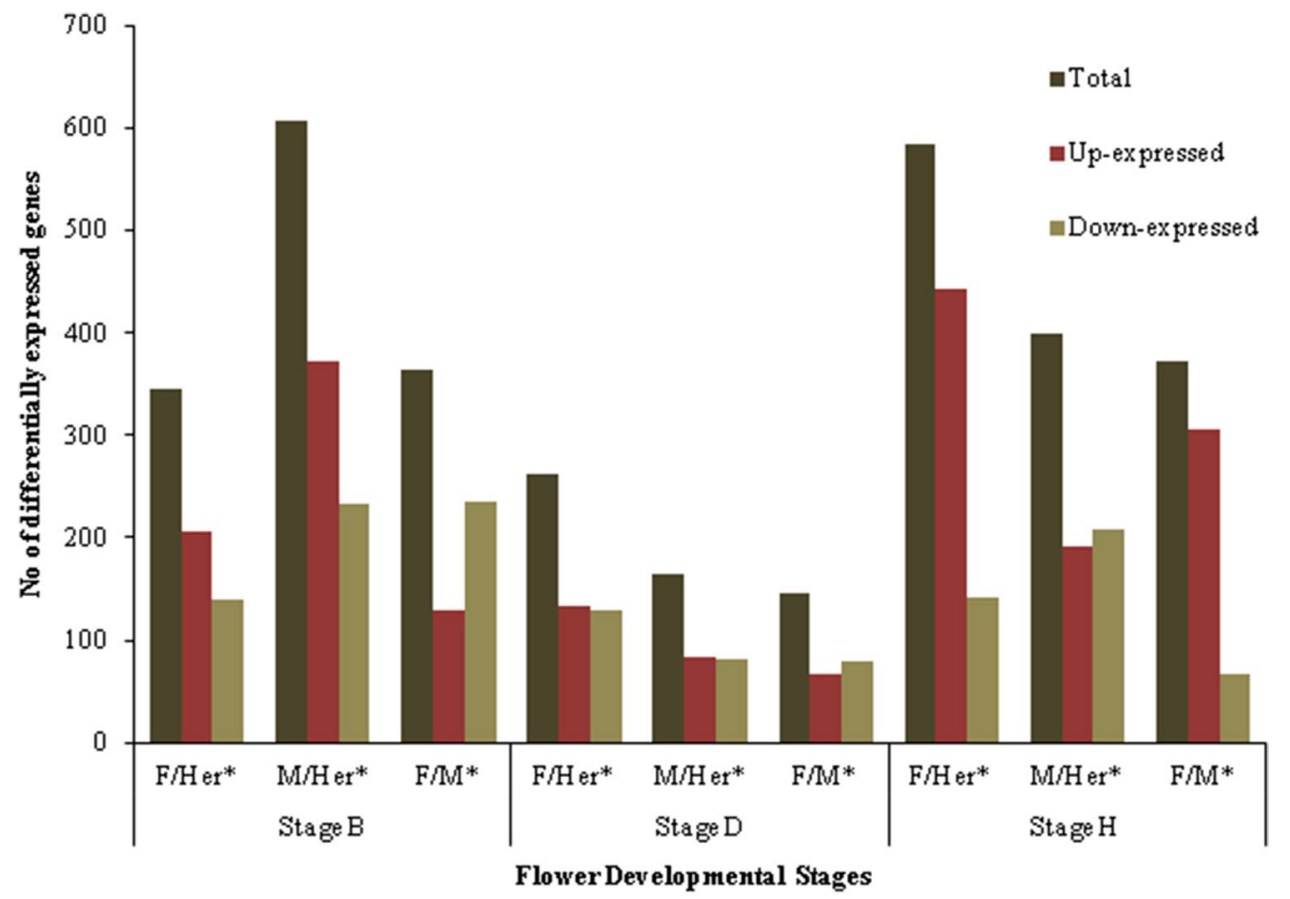

429 DEGs in male flower at developmental stage B (298 upexpressed and 131 down-expressed) (Fig. 8a). Differences between male and female flowers are most visible at developmental stage B, where the female has 272 differential expressed genes (77 up-expressed and 195 down-expressed) (Fig. 8a). However, the differences between female and hermaphrodite flowers are most evident in stage H, where 273 genes are differentially expressed (203 up-expressed and 70 down-expressed) (Fig. 8a). The analysis of the transcripts that have no correspondence in any database show differential expression between flower developmental stages and flower types (Fig. 8b). It allowed to identify a decrease in the number of these differentially expressed transcripts during flower development with $V$. v. sylvestris presenting the largest number of up-expressed transcripts when compared to $V$. v. vinifera (Fig. $8 \mathrm{~b}$ ). These novel sequences could be ancestral genes, targets for genome evolution (Chen et al. 2013). Some of them are still shared between the wild type ( $V$. v. sylvestris) and the domesticated grapevine while gene diversification proceeds until a new gene function evolves. New genes could arise by genome/gene duplication or by retroelements that transcribed through an RNA intermediate (Chen et al. 2013; Senerchia et al. 2013; Zou et al. 2009). The blast against the Repbase (http://www.girinst.org/), a database of mobile elements revealed homologies with mobile elements: transposon sequences (30), LTR retrotransposon (38) and Non-LTR retrotransposon (12) (Table 3). We cannot discard the possibility that these mobile elements are players in plant sex, modulating the genome in order specify the sex.

\section{Transcription factors}

The DEG analysis reveals 36 differentially expressed genes annotated as Transcription Factors (Online Resource Table 1). One transcription factor with strong differential expression is the gene VIT 201s0127g00860 annotated as TRANSCRIPTION FACTOR ABORTED MICROSPORES$L I K E$ (AMS), which shows no expression in any sample until the inflorescence reaches the developmental stage $\mathrm{H}$. In this stage, VIT 201s0127g00860 is strongly expressed in the female inflorescence with differential expression relative to male and hermaphrodite inflorescences. The gene VIT_212s0057g00440 is a GCN5 N-acetyltransferase (GNAT) and could be associated to the transference of an acetyl group, from acetyl coenzyme A (CoA) to a substrate. However, no effective function has been associated to this gene yet. This transcription factor has a basal expression on all samples, but in female inflorescences its action seems to be crucial in flower developmental stage $H$, as showed by the higher expression when compared with male and hermaphrodite samples (Online Resource Table 1). The transcription factor VIT 205s0029g00130 annotated as High mobility group (HMG1) has an opposite expression pattern, with no expression in female plants at developmental stage $\mathrm{H}$ and higher expression in male and hermaphrodite inflorescences.

The MADS-box gene family is a large and important group that promotes changes in gene expression pathways acting as master regulators of developmental switches to define the diversity of floral organs, and to control organ differentiation 
Fig. 7 DEGs between two flower types in the same developmental stage in ten functional categories. Percentage of DEGs present in the transcriptome relatively to the total number of annotated genes by functional category. DEGs were determined in female and a male sample using the hermaphrodite as reference. To compare female and male the male transcriptome was the reference. Filters used: $\mathrm{FDR}=0.05$ and $-1>\log 2$ (fold change) $>1, F$ female, $M$ male, Her hermaphrodite. $\left({ }^{*}\right)$ Sample used as reference

\section{Number of DEGs}

$\begin{array}{lllllllllll}2.5 \% & 2.0 \% & 1.5 \% & 1.0 \% & 0.5 \% & 0.0 \% & 0.5 \% & 1.0 \% & 1.5 \% & 2.0 \% & 2.5 \%\end{array}$

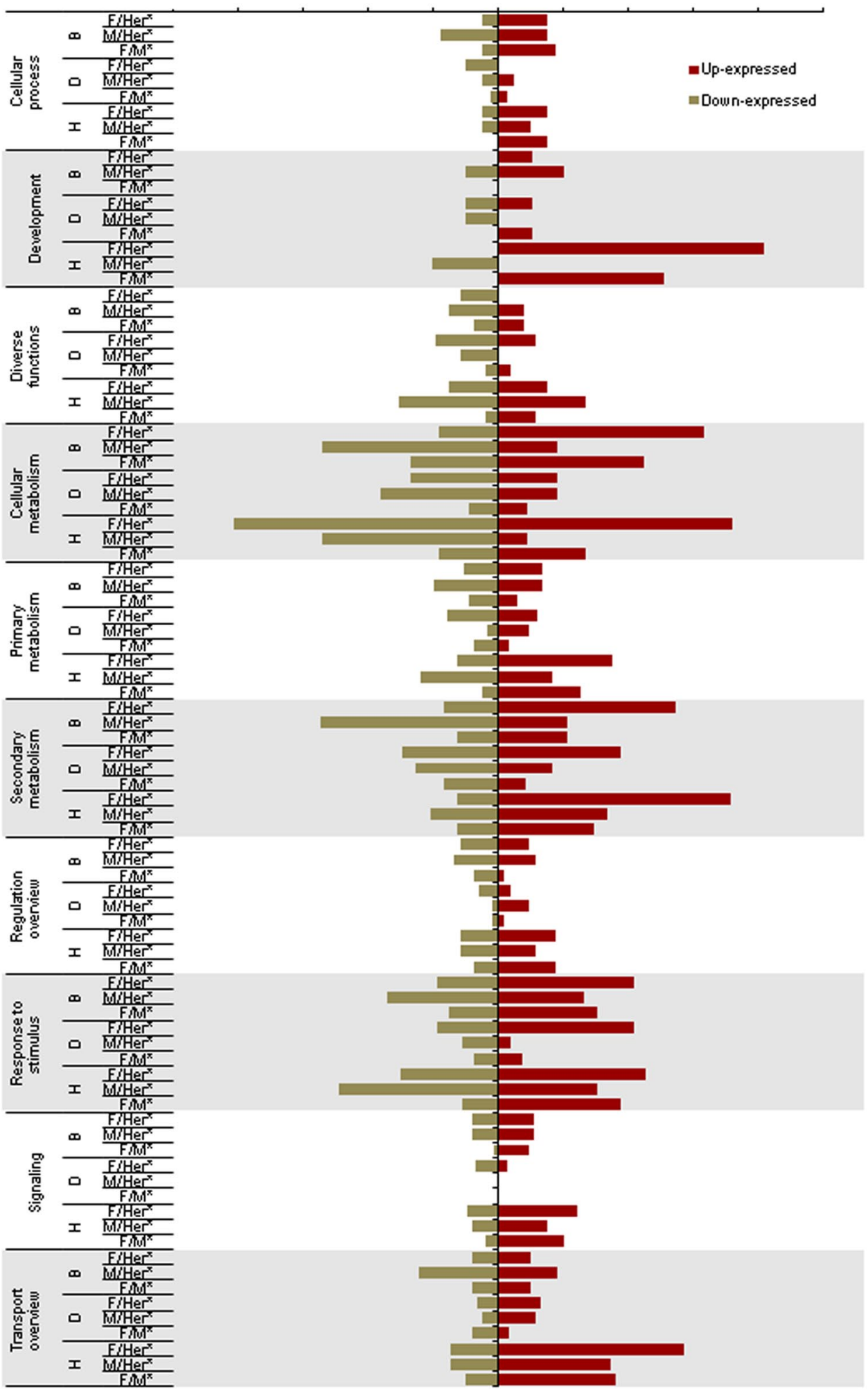

and development (Smaczniak et al. 2012). Three genes annotated as MADS box transcription factors were found to have different behaviors between flower types: AGL16_AGL21 (VIT_218s0001g07900), AGL21 (VIT_200s0211g00180) and SOC1-like (VIT_216s0022g02380). The first two genes (VIT_2 18s0001g07900 and VIT_200s0211g00180) belong to the AGAMOUS clade. The first one is allocated to chromosome 18 and has higher expression in the hermaphrodite 

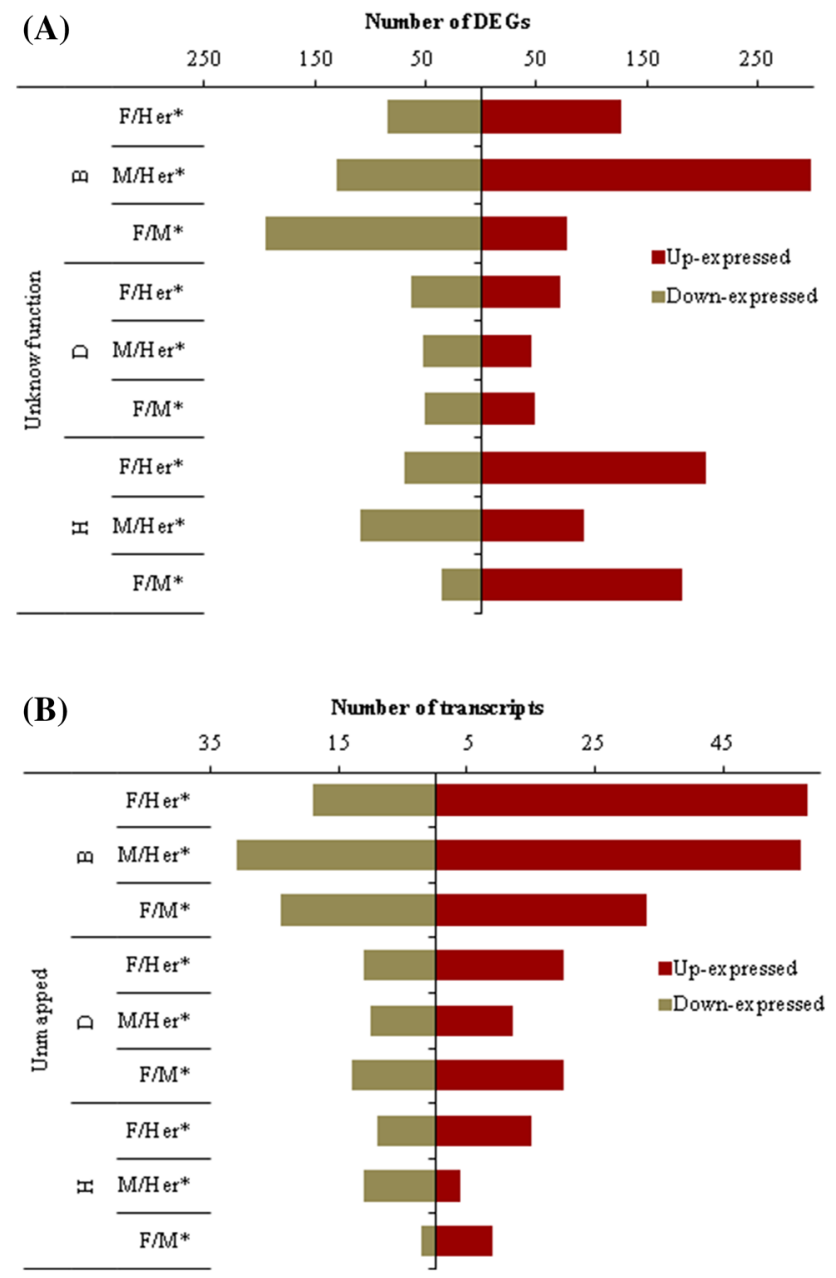

Fig. 8 Overview of differentially expressed genes up and down expressed. a Genes with Unknown function, b Transcripts with no reference in any database. DEGs were determined in female and a male sample using the hermaphrodite as reference. To compare female and male the male transcriptome was the reference. Filters used: $\mathrm{FDR}=0.05$ and $-1>\log 2$ (fold change) $>1, F$ female, $M$ male, $\mathrm{Her}$ hermaphrodite. $(*)$ Sample used as reference

Table 3 Fragments of mobile elements present in sequences with no homologies in any database

\begin{tabular}{llc}
\hline Repeat class & Sequences & $\begin{array}{l}\text { Length } \\
(\mathrm{bp})^{\mathrm{a}}\end{array}$ \\
\hline DNA transposon & 30 & 3255 \\
LTR retrotransposon & 38 & 2601 \\
Non-LTR retrotransposon & 12 & 811 \\
\hline
\end{tabular}

${ }^{a}$ Length; number of the total base pairs present in all sequences

inflorescence when compared with female and male in flower development stage $\mathrm{H}$ and with male developmental stage B. The second one (VIT_200s0211g00180) still belongs to the chromosome Unknown and is highly expressed in male stage B when compared with the same stage in the female inflorescence. Both may be alleles of
$A G L 21$. It is known that $A G L 21$ is involved in root development and are influenced by hormone signals (Yu et al. 2014). Its expression in several stages of Vitis inflorescence development and flower types suggest a possible role in sex specification. Another MADS-box gene is the SOC1-like gene (VIT_216s0022g02380) that has differential expression in female developmental stage B when compared with the hermaphrodite at the same stage. SOCl gene plays an important role in flower development, acting as an integrator of signals from different flowering pathways (Lee and Lee 2010; Zhong et al. 2012). In Vitis, VviSOC1 transcript was found in buds and vegetative organs (Díaz-Riquelme et al. 2009); however, its early expression in inflorescences of female Vitis plants when compared with the hermaphrodite indicates that, directly or indirectly, $V v i S O C l$ is responsible for intrinsic characteristics of the female phenotype.

\section{Hormones and sex specification}

The development of a flower is intrinsically connected to the reception of numerous environmental input signals that coordinate the expression of many genes and interaction of their products.

At first place, the floral meristem identity genes activate floral organ identity genes, whose expression pattern is required for the developmental fate of floral organ primordia (Wellmer et al. 2014).

Numerous hormones including auxin, ethylene, jasmonates, gibberellins (GA) and abscisic acid (ABA) affect floral development (Golenberg and West 2013). DEGs involved in hormone biosynthesis and metabolism could correspond to players in the regulation of flower development as was the case for our data in stage $\mathrm{B}$ and $\mathrm{H}$ of the three types of flowers (Table 4; Online Resource Table 2).

Regarding the phytohormone auxin, in the early stage B there is one gene up (VIT_212s0134g00230) and one gene down-expressed (VIT_209s0002g01340) in female, and two genes down-expressed male flowers (VIT_207s0104g00800 and VIT_205s0049g00460) when compared with the hermaphrodite transcriptome. The later stage $\mathrm{H}$ only has upexpressed genes in female (VIT_212s0059g00610 and VIT_201s0150g00300) and male (VIT_201s0150g00300) when compared with the hermaphrodite. Although, we could not assign one unique function to these genes it is not to exclude the hypothesis that the genes involved in the auxin biosynthesis in Vitis could have a putative role in masculinizing or feminizing the flower, although such effects seem to depend on the Vitis gender and the developmental flower stage (Table 4; Online Resource Table 2).

Genes involved in ethylene signaling are down-expressed in female (VIT_204s0023g02820) compared to male in stage B, and up-expressed in male (VIT_213s0084g00080 and VIT_218s0089g01030) when compared to the 


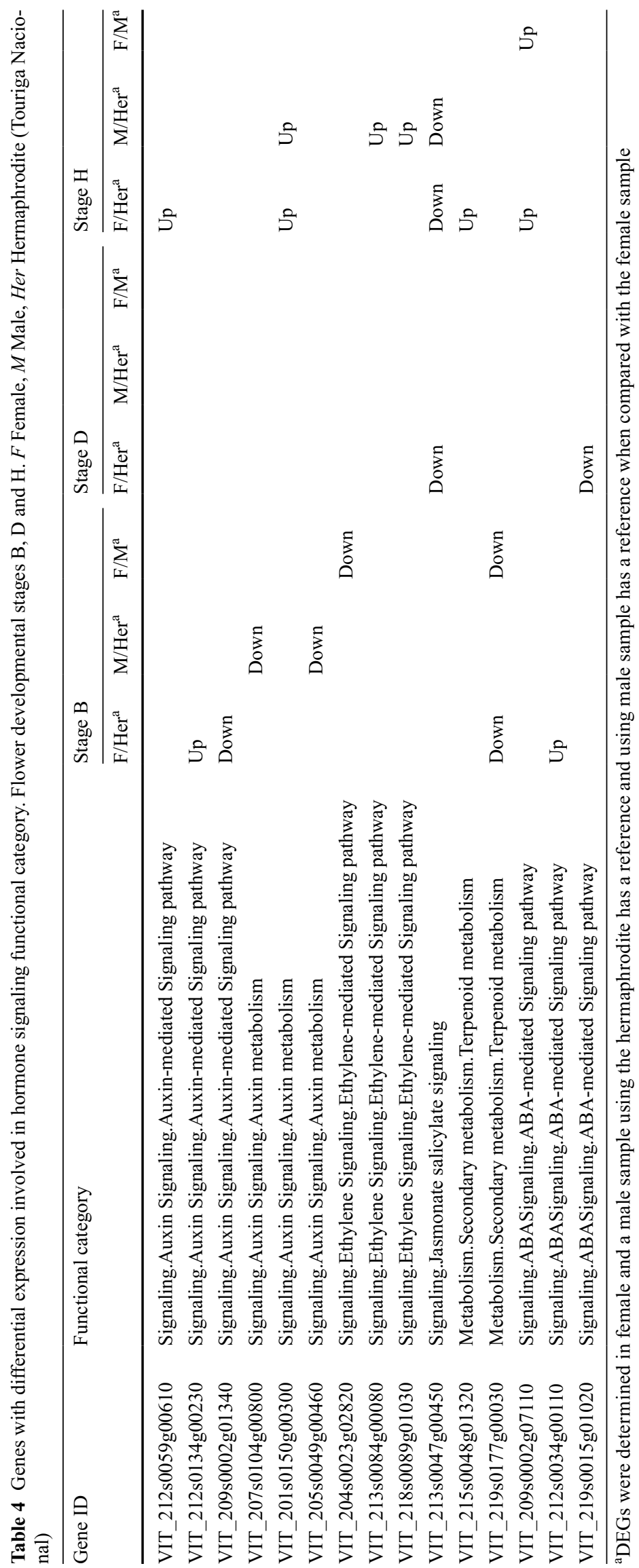


hermaphrodite in stage $\mathrm{H}$. This fact points for a possible masculinizing effect of ethylene in Vitis male plants (Table 4; Online Resource Table 2).

Other genes like 1-aminocyclopropane-1-carboxylate synthase (ACS) involved in ethylene biosynthetic pathway (Wang et al. 2002) regulates unisexual flower development in cucumber (Knopf and Trebitsh 2006; Yamasaki et al. 2003). Two of these genes are differentially expressed in male and female cucumber flowers with high expression in female flowers (Knopf and Trebitsh 2006). However, this gene family has no differential expression in Vitis which suggest another ethylene regulation in early flower development.

The only differentially expressed gene is VIT_213s0047g00450, which is involved in jasmonate signaling. This gene is down-expressed in female flowers in stages $\mathrm{D}$ and $\mathrm{H}$ when compared to the hermaphrodite, and also in males relatively to the hermaphrodite in stage $\mathrm{H}$ (Table 4; Online Resource Table 2). Jasmonate signaling is required for stamen and pollen maturation as show by Arabidopsis mutants that are defective in the synthesis or signaling of jasmonate present male sterile (Park et al. 2002). Jasmonate biosynthesis genes play many roles in regulating flower development. In our expression data we could not assign this gene to a male sterility function as reported for Arabidopsis, but the fact that it is up-expressed in hermaphrodite flowers in stages D and $\mathrm{H}$ hints for an Unknown function of this gene in this type of flowers (Table 4; Online Resource Table 2).

Genes that code for gibberellins show down-expression in female when compared with hermaphrodite and male flowers in stage B (VIT 219s0177g00030), and upexpression in stage $\mathrm{H}$ when compared to the hermaphrodite (VIT 215s0048g01320). For instance, gibberellins have been shown to be important during stamen development, since Arabidopsis mutants with no gibberellins production have compromised stamen development with reduced pollen fertility (Cheng et al. 2004). Gibberellins can also directly affect $L E A F Y$ expression, a floral meristem gene (Blazquez et al. 1998), as well as the expression of floral organ identity genes, like AGAMOUS (AG), APETALA3 (AP3), and PISTILLATA (PI) (Yu et al. 2004). Although, none of these genes were affected by gibberellins in the three Vitis flower types; however, the two differentially expressed genes in Vitis involved in the gibberellins pathway could act at different stages in flower development through a dual role: a masculinizing effect in stage $\mathrm{B}$ and a possible feminizing action in stage $\mathrm{H}$, as evident by up-expression in female flowers (Table 4; Online Resource Table 2).

Several abscisic acid (ABA) response loci are directly involved in controlling flowering time (Kurup et al. 2000), or indirectly involved in meristem function (Kuhn et al. 2007; Lu and Fedoroff 2000). In our data ABA related genes were up-expressed in stage B (VIT 212s0034g00110) and in stage H (VIT_209s0002g07110) and down-expressed in stage D (VIT_219s0015g01020) of female flowers when compared to the hermaphrodite. Genes involved in ABA metabolism may act in the development of stamens in $V$. $v$. sylvestris female plant as it does in tomato (Sekhar and Sawhney 1991).

The differential expression of genes related to hormone biosynthesis seems to be associated to the timing of flower formation in different flower types, rather than with the development of the specific structures of each flower type.

\section{The sex chromosome?}

A region in chromosome 2 spawning from 4,907,434 to $5,037,597 \mathrm{bp}$ has been considered as important for sex determination in $V$. v. vinifera, since it presents a duplication of $122 \mathrm{bp}$ inserted in the gene ADENINE PHOSPHORIBOSYL TRANSFERASE (APT) that acts as a genetic marker for differentiating female from male and hermaphrodite plants (Fechter et al. 2012). More recently, a new study enlarged the region from the marker VVIB23 at 4,781,551 to 5,037,597 bp and found several transcripts with XY type polymorphism (Picq et al. 2014). Considering the importance of this region for sex determination, it was given special attention to the transcripts mapped to this region. It must be stressed that the $12 \mathrm{X} \_$v2.1 annotation several transcripts described in previous works as relevant for sex determination in Vitis are absent from chromosome 2: ETHYLENE OVERPRODUCER LIKE 1 (ETOI) is located in chromosome Unknown (VIT 200s0233g00090) in the Vitis database, ADENINE PHOSPHORIBOSYL TRANSFERASE $(A P T)$ is also located in the chromosome Unknown (VIT 200s1847g00010), and PHOSPHATIDIC ACID PHOSPHATASE (PAP2) is located in the chromosome 12 (VIT 212s0057g01540) (Table 5). In addition, in the 12X_v2.1 annotation, a new gene (VIT_202s0154g00230) was allocated to chromosome 2 between 5,036,984 and 5,037,952 bp at the end of the sex determination region with a sequence annotated as Pinus taeda anonymous locus. This newly allocated Pinus taeda anonymous locus did not have a matching transcript in our assembly. Also several other genes did not have a matching transcript, for example, out of the four FLAVIN-CONTAINING MONOOXYGENASE (FMO) genes, only one was mapped and had its expression quantified (VIT 202s0154g00190) (Table 5). Moreover, in the $4,781,551$ to $5,037,597$ bp regions, several other genes did not have a matching transcript in this assembly. The region spawning from 4,851,659 to 4,875,700 bp harbors four transcripts, including an ABNORMAL FLOWER ORGANS gene (VIT_202s0154g00070), that when blasted with TAIR database (https://www.arabidopsis.org/) retrieves a YABBY transcription factor family transcript. Also without a matching 


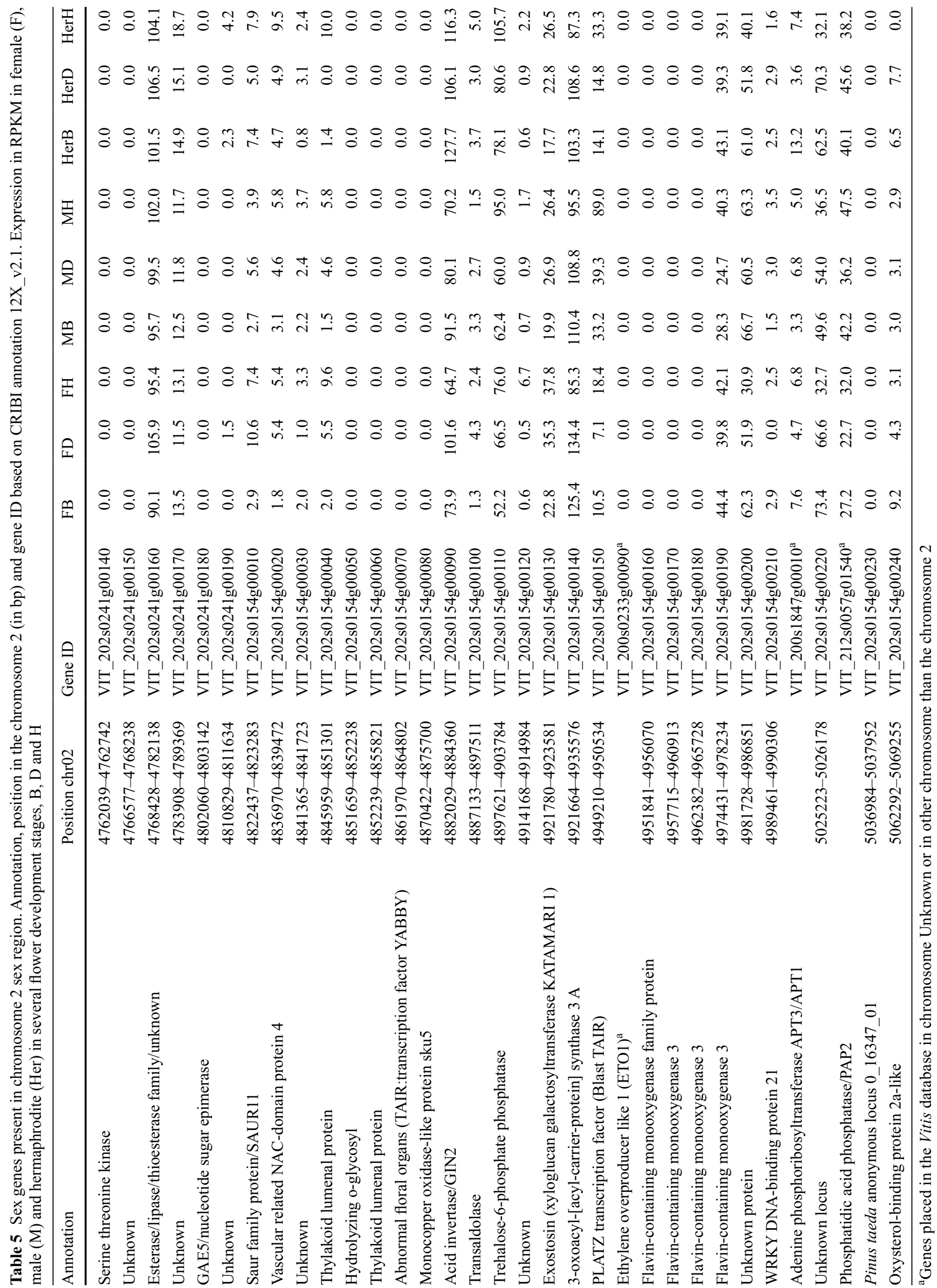


transcript in our assembly and, therefore, no quantifiable expression value (Table 5). The ETO1 absent in the $12 \mathrm{X}_{-}$ v2.1 does not match to any transcript in the assembly, even before filters were applied.

More recently, by using a new heterozygous mapping strategy, it was possible to include five scaffolds from the chromosome Unknown into the chromosome 2, surrounding scaffold_0154 of this chromosome (Fechter et al. 2012; Hyma et al. 2015). Considering the genes comprising these scaffolds, only VIT_200s0229g00190 was differentially expressed in female inflorescences in development stage $\mathrm{D}$, which displays a higher expression value when compared with male and hermaphrodite at the same developmental stage. Also, scaffolds_1344 and scaffold_1682, which comprise a total of three genes (VIT_200s 1344g00010, VIT_200s1682g00010 and VIT_200s1682g00020) were also placed in the sex region of chromosome 2 (Hyma et al. 2015). However, none of these three genes were expressed in our de novo assembly.

Admitting that this region of chromosome 2 is important for Vitis sex determination, it would be expected to find disparities in transcript expression between the different flower types. In the DEG analysis none of the genes that comprise this region was differently expressed. However, flower development is known to involve a myriad of genes and pathways that could interact with other genes for sex specification. The focus on gene expression revealed that most expression disparities occur between development stages, although, few stand out. From 4,810,829 to 4,811,634 bp there is an Unknown transcript (VIT_202s0241g00190), without any matching transcripts in the male plants in any of the three developmental stages. However, VIT 202s0241g00190 has a matching transcript with low expression at stage $\mathrm{D}$ in female flowers, while it displayed matching transcripts in the hermaphrodite flower at stages $\mathrm{B}$ and $\mathrm{H}$, with expression values ranging from 2 (in stage $B$ ) to 4 (in stage $\mathrm{H}$ ) (Table 5). The gene just downstream of this unknown transcript is annotated as a SAUR FAMILY PROTEIN (SAUR11, VIT_202s0154g00010), which stands out for its expression in female stage $\mathrm{D}$ more than doubling when compared with male and hermaphrodite plants at the same stage. Also, male stage $\mathrm{H}$ expression of this transcript is considerably lower than female and hermaphrodite expression at the same stage.

Further downstream, a TREHALOSE-6-PHOSPHATE PHOSPHATASE (VIT 202s0154g00110) was a prominent gene in a previous study (Picq et al. 2014) by displaying $\mathrm{XY}$ type polymorphism. Although this work does not focus on polymorphisms, an increasing expression was observed across developmental stages in the three flower types with a slight decrease in male flower stage D (Table 5). Another gene regarded as having a $\mathrm{XY}$ type polymorphism is the exostosin family protein locus (VIT_202s0154g00130) (Picq et al. 2014), which has a noticeable higher expression in the female flower compared to the male and hermaphrodite flowers, underlining a potentially interesting gene to study in Vitis sex specification. Two loci downstream of EXOSTOSIN locate a PLATZ transcription factor (VIT_202s0154g00150), whose expression in male flowers is considerably higher than in the other two flower types. Between 5,026,178 and 5,036,984 bp resides the locus of the PHOSPHATIDIC ACID PHOSPHATASE (PAP2, VIT_212s0057g01540) (Table 5). In the previous Vitis vinifera annotation this gene was allocated to chromosome 2, however in Vitis reference annotation (12X_v2.1) it is located in the chromosome 12. This is an interesting locus, since its expression in early developmental stages is doubled in both male and hermaphrodite flowers when compared to female flowers, suggesting a possible role in sex determination.

\section{Conclusion}

We carried out a de novo assembly of the transcriptome of three flower developmental stages in three Vitis flower types. This new assembly detected novel areas of transcription, mapping to regions currently not annotated as gene models (intra and intergenic regions) that may represent novel genes. It also suggests, during early Vitis flower development that there is active transcription of previously unannotated genomic loci bioinformatic analysis identified 133 transcripts specific to $V$. v. sylvestris and 516 transcripts differentially expressed among the three flower types, when the three developmental stages were merged. Additionally, some of these regions are differentially expressed during Vitis flower development and may represent novel candidate loci involved in sex establishment such non-coding RNAs, repetitive sequences, mobile elements or miRNAs. Although the specific role of hormones in Vitis sex establishment and flower development has not yet been analyzed in depth, our data show differential gene expression during flower development of genes that control hormone behavior among the three Vitis flower types. Also, analyzing the region of chromosome 2 which has been considered relevant for Vitis sex specification we found that several genes reported as part of that region are still located in chromosome Unknown, suggesting that the latest Vitis annotation (12X_v2.1) needs further confirmation. However, the expression of some of those genes was not detected in the development stages covered by our study. Nevertheless, other genes in the same region displayed interesting expression patterns between the three flower types and could be involved in sex establishment in Vitis.

Acknowledgments This work was supported by the funded project PTDC/AGR-GPL/119298/2010 from Fundação para a Ciência e 
Tecnologia (FCT, Portugal). MRocheta, JLCoito, JCunha, MCosta, MRamos and HGSilva are supported by FCT fellowships SFRH/ BPD/64905/2009，SFRH/BD/85824/2012，SFRH/BPD/74895/2010, $\mathrm{SFRH} / \mathrm{BSAB} / 113781 / 2015, \mathrm{SFRH} / \mathrm{BD} / 110274 / 2015$ and $\mathrm{SFRH} /$ $\mathrm{BD} / 111529 / 2015$, respectively. We are also grateful to Eng. EirasDias, curator from Portuguese Ampelographic Collection (property of Instituto Nacional de Investigação Agrária e Veterinária, Dois Portos) where sampling was performed, for the collaboration in this work allowing the access to the Vitis collection.

Author contributions MR, MNJR and JLC conceived and designed the experiments. MNJR, JLC and MR performed the experiments. MJNR, JLC, MR, JF and OSP analyzed the data. MNJR, JLC, HGS, MMRC and MR wrote the paper. PGA revised and correct the manuscript. SA read and discussed the manuscript. JC established Vitis vinifera sylvestris collection and collected plant tissues samples. All authors read and approved the final manuscript.

\section{Compliance with ethical standards}

Conflict of interest The authors declare that the research was conducted in the absence of any commercial or financial relationships that could be construed as a potential conflict of interest.

\section{References}

Altschul SF, Madden TL, Schaffer AA, Zhang J, Zhang Z, Miller W, Lipman DJ (1997) Gapped BLAST and PSI-BLAST: a new generation of protein database search programs. Nucleic Acids Res 25:3389-3402

Ameur A, Zaghlool A, Halvardson J, Wetterbom A, Gyllensten U, Cavelier L, Feuk L (2011) Total RNA sequencing reveals nascent transcription and widespread co-transcriptional splicing in the human brain. Nat Struct Mol Biol 18:1435-1440. doi:10.1038/ nsmb. 2143

Aukerman MJ, Sakai H (2003) Regulation of flowering time and floral organ identity by a microRNA and its APETALA2-like target genes. Plant Cell 15:2730-2741. doi:10.1105/tpc.016238

Baggiolini M (1952) Les stades repères dans le developpement annuel de la vigne et leur utilisation pratique. Rev Romande Agric Vitic Arbor 8:4-6

Battilana J, Lorenzi S, Moreira FM, Moreno-Sanz P, Failla O, Emanuelli F, Grando MS (2013) Linkage mapping and molecular diversity at the flower sex locus in wild and cultivated grapevine reveal a prominent SSR haplotype in hermaphrodite plants. Mol Biotechnol 54:1031-1037

Blazquez MA, Green R, Nilsson O, Sussman MR, Weigel D (1998) Gibberellins promote flowering of Arabidopsis by activating the LEAFY promoter. Plant Cell 10:791-800

Bondino HG, Valle EM (2009) A small intergenic region drives exclusive tissue-specific expression of the adjacent genes in Arabidopsis thaliana. BMC Mol Biol 10:95. doi:10.1186/1471-2199-10-95

Boualem A et al (2008) A conserved mutation in an ethylene biosynthesis enzyme leads to andromonoecy in melons. Science 321:836-838. doi:10.1126/science. 1159023

Browse J (2009) Jasmonate: preventing the maize tassel from getting in touch with his feminine side Sci Signal 2(59):pe9. doi:10.1126/ scisignal.259pe9

Celaj A, Markle J, Danska J, Parkinson J (2014) Comparison of assembly algorithms for improving rate of metatranscriptomic functional annotation. Microbiome 2:39. doi:10.1186/2049-2618-2-39

Chen S, Krinsky BH, Long M (2013) New genes as drivers of phenotypic evolution. Nat Rev Genet 14:645-660
Cheng H et al (2004) Gibberellin regulates Arabidopsis floral development via suppression of DELLA protein function. Development 131:1055-1064. doi:10.1242/dev.00992

Da Silva C et al (2013) The high polyphenol content of grapevine cultivar tannat berries is conferred primarily by genes that are not shared with the reference genome. Plant Cell 25:4777-4788. doi: $10.1105 /$ tpc. 113.118810

Dalbó MA, Ye GN, Weeden NF, Steinkellner H, Sefc KM, Reisch BI (2000) A gene controlling sex in grapevines placed on a molecular marker-based genetic map. Genome 43:333-340

de Montaigu A, Toth R, Coupland G (2010) Plant development goes like clockwork. Trends Genet 26:296-306. doi:10.1016/j. tig.2010.04.003

Dellaporta SL, Calderon-Urrea A (1994) The sex determination process in maize. Science 266:1501-1505

Díaz-Riquelme J, Lijavetzky D, Martínez-Zapater JM, Carmona MJ (2009) Genome-wide analysis of MIKCC-type MADS box genes in grapevine. Plant Physiol 149:354-369

Durand B, Durand R (1991) Sex determination and reproductive organ differentiation in Mercurialis. Plant Sci 80:49-65. doi:10.1016/0168-9452(91)90272-A

Falgueras J, Lara AJ, Fernández-Pozo N, Cantón FR, Pérez-Trabado G, Claros MG (2010) SeqTrim: a high-throughput pipeline for pre-processing any type of sequence read. BMC Bioinformatics 11:38. doi:10.1186/1471-2105-11-38

Fechter I, Hausmann L, Daum M, Sorensen TR, Viehover P, Weisshaar B, Topfer R (2012) Candidate genes within a $143 \mathrm{~kb}$ region of the flower sex locus in Vitis. Mol Genet Genomics 287:247-259. doi:10.1007/s00438-012-0674-z

Fuchs E, Atsmon D, Halevy AH (1977) Adventitious staminate flower formation in gibberellin treated gynoecious cucumber plants. Plant Cell Physiol 18:1193-1201

Gaidatzis D, Burger L, Florescu M, Stadler MB (2015) Analysis of intronic and exonic reads in RNA-seq data characterizes transcriptional and post-transcriptional regulation. Nat Biotechnol 33:722-729. doi:10.1038/nbt.3269

Gallardo A, Ocete R, López MÁ, Lara M, Riviera D (2009) Assessment of pollen dimorphism in populations of Vitis vinifera $\mathrm{L}$. subsp. sylvestris (Gmelin) Hegi in Spain. VITIS 48:59-62

García RAA, Revilla E (2013) The current status of wild grapevine populations (Vitis vinifera ssp sylvestris) in the Mediterranean Basin. In: Poljuha D, Sladonja B (eds). InTech, pp 51-72. doi: $10.5772 / 52933$

Golenberg EM, West NW (2013) Hormonal interactions and gene gegulation can link monoecy and environmental plasticity to the evolution of dioecy in plants. Am J Bot 100:1022-1037

Guan YF, Huang XY, Zhu J, Gao JF, Zhang HX, Yang ZN (2008) RUPTURED POLLEN GRAIN1, a member of the MtN3/saliva gene family, is crucial for exine pattern formation and cell integrity of microspores in Arabidopsis. Plant Physiol 147:852-863. doi:10.1104/pp.108.118026

Hartwig T et al (2011) Brassinosteroid control of sex determination in maize. Proc Natl Acad Sci USA 108:19814-19819. doi:10.1073/ pnas. 1108359108

Hendriks GJ, Gaidatzis D, Aeschimann F, Grosshans H (2014) Extensive oscillatory gene expression during $C$. elegans larval development. Mol Cell 53:380-392. doi:10.1016/j.molcel.2013.12.013

Hogenesch JB, Ueda HR (2011) Understanding systems-level properties: timely stories from the study of clocks. Nat Rev Genet 12:407-416. doi: $10.1038 / \operatorname{nrg} 2972$

Huijser P, Schmid M (2011) The control of developmental phase transitions in plants. Development 138:4117-4129. doi:10.1242/ dev.063511

Hyma KE et al (2015) Heterozygous mapping strategy (HetMappS) for high resolution genotyping-by-sequencing markers: a case 
study in grapevine. PloS One 10:e0134880. doi:10.1371/journal. pone. 0134880

Jung JH, Seo PJ, Ahn JH, Park CM (2012) Arabidopsis RNA-binding protein FCA regulates microRNA172 processing in thermosensory flowering. J Biol Chem 287:16007-16016

Kapranov P, St Laurent G (2012) Dark matter RNA: Existence, function, and controversy. Front Genet 3:60. doi:10.3389/ fgene. 2012.00060

Kelley JL, Passow CN, Plath M, Arias Rodriguez L, Yee MC, Tobler M (2012) Genomic resources for a model in adaptation and speciation research: characterization of the Poecilia mexicana transcriptome. BMC Genomics 13:652. doi:10.1186/1471-2164-13-652

Knopf RR, Trebitsh T (2006) The female-specific Cs-ACS1G gene of cucumber. A case of gene duplication and recombination between the non-sex-specific 1-aminocyclopropane-1-carboxylate synthase gene and a branched-chain amino acid transaminase gene. Plant Cell Physiol 47:1217-1228

Kuhn JM, Breton G, Schroeder JI (2007) mRNA metabolism of flowering-time regulators in wild-type Arabidopsis revealed by a nuclear cap binding protein mutant, abh1. Plant J 50:1049-1062. doi:10.1111/j.1365-313X.2007.03110.x

Kurup S, Jones HD, Holdsworth MJ (2000) Interactions of the developmental regulator ABI3 with proteins identified from developing Arabidopsis seeds. Plant J 21:143-155

Kwon CS, Chen CB, Wagner D (2005) WUSCHEL is a primary target for transcriptional regulation by SPLAYED in dynamic control of stem cell fate in Arabidopsis. Genes Dev 19:992-1003

Lazarte JE, Garrison SA (1980) Sex modification in Asparagus officinalis L. J Am Soc Hortic Sci 105:691-694

Lebel-Hardenack S, Grant SR (1997) Genetics of sex determination in flowering plants. Trends Plant Sci 2:130-136. doi:10.1016/ S1360-1385(97)01012-1

Lee J, Lee I (2010) Regulation and function of SOC1, a flowering pathway integrator. J Exp Bot 61:2247-2254. doi:10.1093/jxb/erq098

Li B, Dewey CN (2011) RSEM: accurate transcript quantification from RNA-Seq data with or without a reference genome. BMC Bioinformatics 12:323. doi:10.1186/1471-2105-12-323

Li W, Godzik A (2006) Cd-hit: a fast program for clustering and comparing large sets of protein or nucleotide sequences. Bioinformatics 22:1658-1659. doi:10.1093/bioinformatics/btl158

Li B, Fillmore N, Bai Y, Collins M, Thomson JA, Stewart R, Dewey CN (2014) Evaluation of de novo transcriptome assemblies from RNA-Seq data. Genome Biol 15:553. doi:10.1186/ s13059-014-0553-5

Lowe KM, Walker MA (2006) Genetic linkage map of the interspecific grape rootstock cross Ramsey (Vitis champinii) $\times$ Riparia Gloire (Vitis riparia). Theor Appl Genet 112:1582-1592. doi:10.1007/ s00122-006-0264-8

Lu C, Fedoroff N (2000) A mutation in the Arabidopsis HYL1 gene encoding a dsRNA binding protein affects responses to abscisic acid, auxin, and cytokinin. Plant Cell 12:2351-2366. doi:10.1105/ tpc.12.12.2351

Manzano S, Martinez C, Garcia JM, Megias Z, Jamilena M (2014) Involvement of ethylene in sex expression and female flower development in watermelon (Citrullus lanatus). Plant Physiol Biochem 85:96-104. doi:10.1016/j.plaphy.2014.11.004

Marguerit E et al (2009) Genetic dissection of sex determinism, inflorescence morphology and downy mildew resistance in grapevine. Theor Appl Genet 118:1261-1278. doi:10.1007/ s00122-009-0979-4

Moghe GD et al (2013) Characteristics and significance of intergenic polyadenylated RNA transcription in Arabidopsis. Plant Physiol 161:210-224. doi:10.1104/pp.112.205245

Mohawk JA, Green CB, Takahashi JS (2012) Central and peripheral circadian clocks in mammals. Annu Rev Neurosci 35:445-462. doi:10.1146/annurev-neuro-060909-153128
Negi SS, Olmo HP (1966) Sex conversion in a male Vitis vinifera L. by a kinin. Science 152:1624-1625

Orozco-Arroyo G, Vazquez-Santana S, Camacho A, Dubrovsky JG, Cruz-Garcia F (2012) Inception of maleness: auxin contribution to flower masculinization in the dioecious cactus Opuntia stenopetala. Planta 236:225-238. doi:10.1007/ s00425-012-1602-5

Oshlack A, Robinson MD, Young MD (2010) From RNA-seq reads to differential expression results. Genome Biol 11:220. doi:10.1186/ gb-2010-11-12-220

Park JH, Halitschke R, Kim HB, Baldwin IT, Feldmann KA, Feyereisen R (2002) A knock-out mutation in allene oxide synthase results in male sterility and defective wound signal transduction in Arabidopsis due to a block in jasmonic acid biosynthesis. Plant J 31:1-12

Picq S et al (2014) A small XY chromosomal region explains sex determination in wild dioecious $V$. vinifera and the reversal to hermaphroditism in domesticated grapevines. BMC Plant Biol 14:229. doi:10.1186/s12870-014-0229-z

Ponting CP, Belgard TG (2010) Transcribed dark matter: meaning or myth? Hum Mol Genet 19:R162-R168. doi:10.1093/hmg/ddq362

Ramos MJ, Coito JL, Silva HG, Cunha J, Costa MM, Rocheta M (2014) Flower development and sex specification in wild grapevine. BMC Genomics 15:1095. doi:10.1186/1471-2164-15-1095

Ryan PT et al (2015) Patterns of gene expression during Arabidopsis flower development from the time of initiation to maturation. BMC Genomics 16:488. doi:10.1186/s12864-015-1699-6

Schulz MH, Zerbino DR, Vingron M, Birney E (2012) Oases: robust de novo RNA-seq assembly across the dynamic range of expression levels. Bioinformatics 28:1086-1092. doi:10.1093/ bioinformatics/bts094

Sekhar KNC, Sawhney VK (1991) Role of ABA in stamen and pistil development in the normal and solanifolia mutant of tomato (Lycopersicon esculentum). Sex Plant Reprod 4:279-283. doi:10.1007/BF00200548

Senerchia N, Wicker T, Felber F, Parisod C (2013) Evolutionary dynamics of retrotransposons assessed by high-throughput sequencing in wild relatives of wheat. Genome Biol Evol 5:1010 1020. doi:10.1093/gbe/evt064

Smaczniak C, Immink RG, Angenent GC, Kaufmann K (2012) Developmental and evolutionary diversity of plant MADS-domain factors: insights from recent studies. Development 139:3081-3098. doi:10.1242/dev.074674

Sultan M et al (2008) A global view of gene activity and alternative splicing by deep sequencing of the human transcriptome. Science 321:956-960. doi:10.1126/science.1160342

Wagner D, Meyerowitz EM (2002) SPLAYED, a novel SWI/SNF ATPase homolog, controls reproductive development in Arabidopsis. Curr Biol 12:85-94

Wang KLC, Li H, Ecker JR (2002) Ethylene biosynthesis and signaling networks. Plant Cell 14:S131-S151

Wang Z, Gerstein M, Snyder M (2009) RNA-Seq: a revolutionary tool for transcriptomics. Nat Rev Genet 10:57-63. doi:10.1038/nrg2484

Wellmer F, Graciet E, Riechmann JL (2014) Specification of floral organs in Arabidopsis. J Exp Bot 65:1-9. doi:10.1093/jxb/ert385

Wu MF et al (2012) SWI2/SNF2 chromatin remodeling ATPases overcome polycomb repression and control floral organ identity with the $L E A F Y$ and SEPALLATA3 transcription factors. Proc Natl Acad Sci USA 109:3576-3581

Yamasaki S, Fujii N, Takahashi H (2003) Characterization of ethylene effects on sex determination in cucumber plants. Sex Plant Reprod 16:103-111

Yu H, Ito T, Zhao Y, Peng J, Kumar P, Meyerowitz EM (2004) Floral homeotic genes are targets of gibberellin signaling in flower development. Proc Natl Acad Sci USA 101:7827-7832. doi:10.1073/pnas.0402377101 
Yu LH, Miao ZQ, Qi GF, Wu J, Cai XT, Mao JL, Xiang CB (2014) MADS-box transcription factor $A G L 21$ regulates lateral root development and responds to multiple external and physiological signals. Mol Plant 7:1653-1669. doi:10.1093/mp/ssu088

Zenoni S et al (2010) Characterization of transcriptional complexity during berry development in Vitis vinifera using RNA-Seq. Plant Physiol 152:1787-1795. doi:10.1104/pp.109.149716

Zerbino DR (2010) Using the Velvet de novo assembler for short-read sequencing technologies. Curr Protoc Bioinformatics 11(5):1-13. doi:10.1002/0471250953.bi1105s31
Zerbino DR, Birney E (2008) Velvet: algorithms for de novo short read assembly using de Bruijn graphs. Genome Res 18:821-829. doi:10.1101/gr.074492.107

Zhong X, Dai X, Xv J, Wu H, Liu B, Li H (2012) Cloning and expression analysis of GmGAL1, SOC1 homolog gene in soybean. Mol Biol Rep 39:6967-6974. doi:10.1007/s11033-012-1524-0

Zou J, Gong H, Yang T-J, Meng J (2009) Retrotransposons: a major driving force in plant genome evolution and a useful tool for genome analysis. J Crop Sci Biotechnol 12:1-8. doi:10.1007/ s12892-009-0070-3 